\title{
From a Polynomial Riemann Hypothesis to Alternating Sign Matrices
}

\author{
Ömer Eğecioğlu* \\ Department of Computer Science \\ University of California, Santa Barbara CA 93106 \\ omer@cs.ucsb.edu \\ Timothy Redmond \\ Network Associates Inc., \\ 3965 Freedom Circle, Santa Clara, CA 95054 \\ redmond@best.com \\ Charles Ryavec \\ College of Creative Studies, \\ University of California, Santa Barbara CA 93106 \\ ryavec@math . ucsb.edu \\ Submitted: March 27, 2001; Accepted: October 24, 2001. \\ MR Subject Classifications: 05E35, 11M26, 12D10
}

\begin{abstract}
This paper begins with a brief discussion of a class of polynomial Riemann hypotheses, which leads to the consideration of sequences of orthogonal polynomials and 3-term recursions. The discussion further leads to higher order polynomial recursions, including 4-term recursions where orthogonality is lost. Nevertheless, we show that classical results on the nature of zeros of real orthogonal polynomials (i. e., that the zeros of $p_{n}$ are real and those of $p_{n+1}$ interleave those of $p_{n}$ ) may be extended to polynomial sequences satisfying certain 4 -term recursions. We identify specific polynomial sequences satisfying higher order recursions that should also satisfy this classical result. As with the 3-term recursions, the 4 -term recursions give rise naturally to a linear functional. In the case of 3-term recursions the zeros fall nicely into place when it is known that the functional is positive, but in the case of our 4-term recursions, we show that the functional can be positive even when there are non-real zeros among some of the polynomials. It is interesting, however, that for our 4-term recursions positivity is guaranteed when a certain real parameter $C$ satisfies $C \geq 3$, and this is exactly the condition of our result that guarantees the zeros have the aforementioned interleaving property. We conjecture the condition $C \geq 3$ is also necessary.

Next we used a classical determinant criterion to find exactly when the associated linear functional is positive, and we found that the Hankel determinants $\Delta_{n}$ formed from the sequence of moments of the functional when $C=3$ give rise to the initial values of the integer sequence $1,3,26,646,45885, \cdots$, of Alternating Sign Matrices (ASMs) with vertical symmetry. This spurred an intense interest in these moments, and we give 9 diverse characterizations of this sequence of moments. We then specify these Hankel determinants as
\end{abstract}

\footnotetext{
${ }^{*}$ Supported in part by NSF Grant No. CCR-9821038.
} 
Macdonald-type integrals. We also provide an an infinite class of integer sequences, each sequence of which gives the Hankel determinants $\Delta_{n}$ of the moments.

Finally we show that certain $n$-tuples of non-intersecting lattice paths are evaluated by a related class of special Hankel determinants. This class includes the $\Delta_{n}$. At the same time, ASMs with vertical symmetry can readily be identified with certain $n$-tuples of osculating paths. These two lattice path models appear as a natural bridge from the ASMs with vertical symmetry to Hankel determinants.

\section{Contents}

1 Introduction $\quad 3$

2 The 3-Conjecture $\quad 6$

3 The 6-Conjecture $\quad 11$

4 Moments $\quad 12$

5 Very Special Hankel Determinants $\quad 22$

6 Positivity is Insufficient $\quad 24$

7 Certain Macdonald-type Integrals $\quad 25$

8 Equivalent forms for $\Delta_{n} \quad 28$

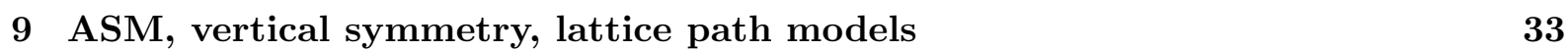

10 Path Interpretations \& Hankel Determinants $\quad 37$

11 Higher Order $\Delta_{n} \quad 44$

12 Epilogue $\quad 45$

13 APPENDIX I (Derivation of the 4-term recursion) 48

14 APPENDIX II (Renormalized 4-term recursion) 50

THE ELECTRONIC JOURNAL OF COMBINATORICS 8 (2001), \#R36 2 


\section{Introduction}

Let $g(x)$ be a real polynomial and $T[g](s)$ be the polynomial defined linearly on basis elements by

$$
\begin{aligned}
T[1](s) & =1 \\
T\left[x^{n}\right](s) & =s(s+1) \cdots(s+n-1) / n ! .
\end{aligned}
$$

The transformation $T$ can be viewed in terms of the complex integral transform

$$
T[g](s) \frac{\pi}{\sin (\pi s)}=\int_{0}^{1} x^{s}(1-x)^{1-s} g(x) \frac{d x}{x(1-x)} .
$$

Furthermore if $g(x)=g(1-x)$ then

$$
T[g](s)=T[g](1-s) .
$$

Especially interesting would be those cases in which $T[g](s)$ satisfies, additionally a Riemann hypothesis; i.e., in those cases in which the zeros $\rho=\beta+i \gamma$, satisfy $\beta=\frac{1}{2}$.

Redmond has recently given an analytic proof that shows that whenever the polynomial $g$ satisfies a Riemann hypothesis, then so does the $T$-transform $T[g]$. Although this result does not include those situations where the polynomial $g$ does not satisfy a Riemann hypothesis, but $T[g](s)$ does, he has been able to generalize $g \in R h \Rightarrow T[g] \in R h$ to entire $g$ of order 1 (see [9]).

As an example, his result shows that the polynomials

$$
T\left[(x+r)^{n}+(1-x+r)^{n}\right](s)
$$

satisfy a Riemann hypothesis for all $n>0$ and all values of the real parameter $r$. A substantial amount of numerical evidence indicates that a great deal more is true and we give two examples to illustrate the important phenomena of positivity and interlacing that are inaccessible by analytic methods.

First, when $r>0$, the polynomials

$$
T\left[(x+r)^{n}\right]\left(w+\frac{1}{2}\right)=\sum_{i, j \geq 0} c_{i j} w^{i} r^{j}
$$

can be shown to have the positivity property that all the coefficients $c_{i j}$ are non-negative, which can be used [4] to show that the $w$-zeros of $T\left[(x+r)^{n}\right]\left(w+\frac{1}{2}\right)$ are negative when $r>0$.

Using this positivity result and other results, together with known parts of the standard theory of 3-term polynomial recursions, Eğecioğlu and Ryavec [4] were able to show in a completely different way that for all $n>0$ and all real values of the parameter $r$, the polynomials given in (2) satisfy a Riemann hypothesis. The proof techniques here have implications that are the subject matter of this paper.

After having disposed of what might be termed The Linear Case by these alternative techniques, it seemed natural to consider the Quadratic Case; i. e., to consider the zeros of

$$
P_{n}(s, r)=T\left[(x(x-1)+r)^{n}\right](s),
$$

THE ELECTRONiC JOURNAL OF COMBINATORICS 8 (2001), \#R36 
for values of the parameter $r$ satisfying $r \geq \frac{1}{4}$. Here again Redmond's result shows that the $P_{n}(s, r)$ satisfy a Riemann hypothesis, but it is again likely that much more is true as we indicate.

The polynomials $P_{n}(s, r)$ generate real polynomials

$$
P_{n}\left(\frac{1}{2}+i t, r\right)
$$

in $t^{2}$, so that if we put $u=-t^{2}$ and set

$$
p_{n}(u, r)=P_{n}\left(\frac{1}{2}+i t, r\right)
$$

then the $p_{n}$ satisfy a 4 -term recursion. Numerical data indicates that for each $r \geq \frac{1}{4}$, the $u$-zeros of $p_{n+1}(u, r)$ are negative and interlace the $u$-zeros of $p_{n}(u, r)$. We have called this assertion the Quadratic Polynomial Riemann hypothesis. Moreover, the data also supports the assertion that a positivity result (like the result established in the Linear Case) holds in the Quadratic Case; i. e., that if

$$
p_{n}\left(u, R+\frac{1}{4}\right)=\sum_{i, j \geq 0} c_{i, j} u^{i} R^{j},
$$

then the nonzero coefficients $c_{i, j}$ are positive. If true, this would show that if the roots of the $p_{n}(u, r)$ are real, then they are negative for $r \geq \frac{1}{4}$, which is equivalent to $P_{n}(s, r) \in R h$.

We cannot provide a proof of the polynomial Riemann hypothesis in the Quadratic Case. If the hypothesis is correct, it is interesting when considered within the framework of the general theory of polynomial recursions.

The new feature in the Quadratic Case is that the $p_{n}(u, r)$ do not satisfy a 3-term recursion for $r>\frac{1}{4}$, but rather a 4-term recursion. Essentially the 3-term theory, on which the Linear Case relies, is based on a notion of orthogonality not available in the consideration of 4 -term recursions. In other words, the standard arguments of the 3-term theory are then too weak to extend to a 4-term theory, and in fact they cannot be extended in any general statement.

Without any existing theory available to tackle the Quadratic Polynomial Riemann hypothesis, we turned to the consideration of renormalized versions of the 4-term recursions satisfied by the $p_{n}$. The recursions for the $p_{n}$ are given in (5) of section 2 . We mention that the term "renormalization" refers to a series of elementary transformations (described in Appendix II) that convert the 4-term polynomial recursions (5) into the 4-term polynomial recursions (6). Renormalization therefore has the effect of condensing the somewhat complicated recursions (5) in the parameters $n$ and $r$ into a relatively simple recursion (6) in the single parameter $C$. This simple recursion identified $C=3$ as a critical value, and led to the formulation of the 3-Conjecture. This conjecture might be viewed as a single asymptotic version of the Quadratic Polynomial Riemann hypothesis, and again, substantial amount of data indicates its truth. On the other hand, this conjecture is readily phrased in two halves, and Redmond was able to prove the most important half, and his proof is included in this paper as Theorem 1. Higher order conjectures are probably true and examples are given.

In a strange twist of fortune, certain determinants $\Delta_{n}$ which are naturally attached to the 3-Conjecture (and which will appear in section 5), open up some very unexpected connections to Alternating Sign Matrices (ASM's). In fact when the sequence of integers 1, 3, 26, 646, 
$45885, \cdots$, first appeared on the screen, our amazement was total. From that point on everything we touched seemed inexorably (and for a time, inexplicably) to generate these integers, and the following table lists some of the many models considered in this paper that are connected via this fascinating sequence. The symbols in the first column will be explained in due course, and

$$
\begin{array}{rccccccc}
n & : & 0 & 1 & 2 & 3 & 4 & \cdots \\
\Delta_{n} & : & 1 & 3 & 26 & 646 & 45885 & \ldots \\
R R(n) & : & 1 & 3 & 26 & 646 & 45885 & \ldots \\
I_{n} & : & 1 & 3 & 26 & 646 & 45885 & \ldots \\
A_{n} & : & 1 & 3 & 26 & 646 & 45885 & \ldots \\
V_{n} & : & 1 & 3 & 26 & 646 & 45885 & \ldots \\
O_{n} & : & 1 & 3 & 26 & 646 & 45885 & \ldots \\
P_{n} & : & 1 & 3 & 26 & 646 & 45885 & \ldots
\end{array}
$$

Figure 1: Different models for $1,3,26,646,45885, \cdots$

we begin with the Robbins-Rumsey sequence,

$$
R R(n)=\prod_{k=0}^{n} \frac{\left(\begin{array}{c}
6 k+4 \\
2 k+2
\end{array}\right)}{2\left(\begin{array}{c}
4 k+3 \\
2 k+2
\end{array}\right)},
$$

listed in [10] as the conjectured counting formula for the number $V_{n}$ of ASM's with vertical symmetry. This conjecture (and others) has recently been proved by Kuperberg [6]. In this paper we prove several results and indicate directions for further conjectures. In Theorem 3 (section 7) we show that

$$
\Delta_{n}=I_{n},
$$

where $I_{n}$ is a sequence of values of certain Macdonald-type integrals (see (27), Section 7). In Theorem 4 (section 8 ) we show that

$$
I_{n}=A_{n},
$$

where $A_{n}$ is any one of the sequence of Hankel determinants given in Theorem 4. In Theorem 5 , we show that

$$
A_{n}=R R(n) .
$$

There are two sequences, $O_{n}$ (Definition 1, Section 9) and $P_{n}$ (Definition 2, Section 10), that count two types, respectively, of ensembles of lattice paths. We show in Lemma 2 (section 9) that

$$
V_{n}=O_{n}
$$

and we show in Theorem 6 (section 10) that

$$
A_{n}=P_{n}
$$

THE ELECTRONiC JOURNAL OF COMBINATORICS 8 (2001), \#R36 
A completely different proof of the Robbins-Rumsey conjecture

$$
V_{n}=R R(n)
$$

would follow from a bijection between the lattice paths counted by $O_{n}$ and those counted by $P_{n}$, or equivalently, between the two corresponding families of tableaux described at the end of section 10 .

\section{The 3-Conjecture}

Using (1) we construct the first few polynomials $P_{n}(s, r)$ defined in (3) as

$$
\begin{aligned}
& P_{0}(s, r)=1 \\
& P_{1}(s, r)=\frac{1}{2} s(s-1)+r \\
& P_{2}(s, r)=\frac{1}{24} s^{2}(s-1)^{2}+\left(r-\frac{1}{12}\right) s(s-1)+r^{2} .
\end{aligned}
$$

For $n \geq 2$, it can be shown that the $P_{n}$ satisfy the 4 -term recursion

$$
\begin{aligned}
(2 n+2)(2 n+1) P_{n+1}(s) & =\left[s(s-1)+12 r n^{2}+8 r n+2 r-n^{2}-n\right] P_{n}(s) \\
& -\left[12 r^{2} n^{2}-2 r n^{2}-2 r^{2} n\right] P_{n-1}(s) \\
& +\left[n(n-1)\left(4 r^{3}-r^{2}\right)\right] P_{n-2}(s) .
\end{aligned}
$$

This recursion is derived in Appendix I. The $p_{n}(u)$ of $(4)$ therefore satisfy the recursion

$$
\begin{aligned}
(2 n+2)(2 n+1) p_{n+1}(u) & =\left[-\frac{1}{4}+u+12 r n^{2}+8 r n+2 r-n^{2}-n\right] p_{n}(u) \\
& -\left[12 r^{2} n^{2}-2 r n^{2}-2 r^{2} n\right] p_{n-1}(u) \\
& +\left[n(n-1)\left(4 r^{3}-r^{2}\right)\right] p_{n-2}(u)
\end{aligned}
$$

which, as a tool in proving the Quadratic Polynomial Riemann hypothesis, we found intractable, and we turned to efforts at simplifying the recursion by renormalization. Renormalization is an attempt to see what is happening in the $p_{n}$-recursion (5) for large $n$. We have put the steps in the renormalization into Appendix II and quote here merely the new polynomial recursion that results from the renormalization of the $p_{n}$. Thus we obtained a sequence of polynomials $q_{n}=q_{n}(x)$ with $q_{-2}=q_{-1}=0, q_{0}=1$, and defined thereafter by the recursion

$$
q_{n}=x q_{n-1}-C q_{n-2}-q_{n-3},
$$

where

$$
C=\frac{8 r(6 r-1)}{\left[16 r^{2}(4 r-1)\right]^{\frac{2}{3}}} .
$$

As $r$ runs from $\frac{1}{4}$ to $\infty, C(r)$ is monotone decreasing to 3 , and we find that $C=3$ is a critical value in several important respects. Before we consider the 4-term recursion (6), it will 
be useful to review briefly some of the theory of 3-term recursions (we refer the reader to [3] for details).

Consider a sequence of polynomials $q_{n}(x)$ defined by the 3 -term recursion,

$$
q_{n}=\left(x-c_{n}\right) q_{n-1}-\lambda_{n} q_{n-2},
$$

where $q_{-1}=0, q_{0}=1$ and the $\left\{c_{n}\right\}$ and $\left\{\lambda_{n}\right\}$ are real sequences. There is then a unique linear functional $\mathcal{L}$ on the space of polynomials such that

$$
\begin{aligned}
\mathcal{L}[1] & =\lambda_{1} \\
\mathcal{L}\left[q_{m} q_{n}\right] & =0 m \neq n \\
\mathcal{L}\left[q_{n}^{2}\right] & =\lambda_{1} \lambda_{2} \cdots \lambda_{n+1}
\end{aligned}
$$

It follows that the $\left\{q_{n}\right\}$ is an orthogonal sequence of monic polynomials with respect to $\mathcal{L}$ if the $\lambda_{n} \neq 0$.

The functional $\mathcal{L}$ is said to be positive definite if $\mathcal{L}[p]>0$ for every non-negative, non-zero polynomial $p$. Therefore $\mathcal{L}$ is positive definite if and only if all $\lambda_{n}>0$. In this case, the zeros of the $q_{n+1}$ are real and simple and interlace the zeros of $q_{n}$. Moreover, if we specify the moments of $\mathcal{L}$ by

$$
\mu_{n}=\mathcal{L}\left[x^{n}\right]
$$

(and take $\mu_{0}=\lambda_{1}=1$ ), then $\mathcal{L}$ is positive definite if and only if the associated sequence of Hankel determinants

$$
\Delta_{n}=\Delta_{n}\left[\mu_{i+j}\right]_{0 \leq i, j \leq n}
$$

are positive for $n=0,1, \ldots$

Now if you begin with a sequence of monic polynomials $q_{n}$ defined as in (6) by a 4 -term recursion, then you again get some orthogonality with respect to the functional $\mathcal{L}_{C}$ defined by

$$
\begin{aligned}
\mathcal{L}_{C}[1] & =\mu_{0}=1 \\
\mathcal{L}_{C}\left[x^{n}\right] & =\mu_{n} \\
\mathcal{L}_{C}\left[q_{n}\right] & =0 \quad n \geq 1,
\end{aligned}
$$

which results in

$$
\mathcal{L}_{C}\left[q_{1} q_{3}\right]=0
$$

but not, for example,

$$
\mathcal{L}_{C}\left[q_{2} q_{3}\right]=0 .
$$

Evidently, this loss of orthogonality makes it impossible to transfer directly the arguments of the 3 -term theory to the 4-term situation.

Our first result, the so-called 3-Conjecture, relates to the Quadratic Polynomial Riemann hypothesis and the 4-term recursions (6). We have the following conjecture.

Conjecture 1 (3-Conjecture) The sequence of polynomials $q_{n}, n=1,2, \ldots$, as defined through the 4-term recursion (6) have real zeros if and only if $C \geq 3$. Moreover, when $C \geq 3$, the zeros of $q_{n+1}$ interlace the zeros of $q_{n}$. 
This conjecture is proved in the case that $C \geq 3$. We do not have a proof of the statement that when $C<3$, then there is some $q_{n}$ with some non-real zeros. Numerical evidence for values of $C$ as high as $C=2.9$ gives $n$ with $q_{n}$ having some non-real zeros and indicates that $C=3$ is indeed the critical value.

Theorem 1 If $C \geq 3$ then the polynomials defined by $q_{-2}=q_{-1}=0, q_{0}=1$ and by (6) for $n \geq 1$ have real zeros, and the zeros of $q_{n+1}$ interleave the zeros of $q_{n}$.

Proof The proof breaks down into the following steps:

1. Fix $N$ large and restrict attention to the polynomials. $\left(q_{n}(x)\right)_{0 \leq n<N}$.

2. Show that if $C$ is sufficiently large then the zeros of $\left(q_{n}(x)\right)_{0 \leq n<N}$ are real and interleaved.

3. If for some $C$, the zeros of $\left(q_{n}(x)\right)_{0 \leq n<N}$ are not real and interleaved then as $C$ decreases there must be a transition at some point. At the point of the transition there will be a $k$ with $0<k<N-1$ and a real $x_{0}$ such that $q_{k}\left(x_{0}\right)=q_{k+1}\left(x_{0}\right)=0$.

4. Fix $C$ and $x_{0}$ to be this transition point and assume that $C \geq 3$. Let $t_{1}, t_{2}, t_{3}$ be the roots of the polynomial,

$$
t^{3}-x_{0} t^{2}+C t+1=0
$$

5. Show that two of the roots must be equal.

6. Dispose of the double root case.

7. Dispose of the triple root case.

\section{Large $C$ case and the transition}

Fix $N>0$. We first need to show that for sufficiently large $C$ the roots of the first $N$ polynomials are real and interleaved. We do this by scaling and showing that after scaling and normalization the $q_{n}$ are a simple perturbation of orthogonal polynomials. Note that

$$
\frac{q_{n+1}(\sqrt{C} x)}{C^{(n+1) / 2}}=x \frac{q_{n}(\sqrt{C} x)}{C^{n / 2}}-\frac{q_{n-1}(\sqrt{C} x)}{C^{(n-1) / 2}}-\frac{1}{C^{3 / 2}} \frac{q_{n-2}(\sqrt{C} x)}{C^{(n-2) / 2}}
$$

Thus if we define

$$
q_{n}(x)=\frac{q_{n}(\sqrt{C} x)}{C^{n / 2}}
$$

then $q_{n}$ satisfies the following recursion

$$
q_{n+1}(x)=x q_{n}(x)-q_{n-1}(x)-C^{-3 / 2} q_{n-2}(x) .
$$

For large $C$ this is just a perturbation of the recursion

$$
r_{n+1}(x)=x r_{n}(x)-r_{n-1}(x)
$$


which defines a set of orthogonal polynomials. Thus the first set of $N$ polynomials of $q$ can be made arbitrarily close to the first $N$ polynomials $r_{n}(n=0,1,2, \ldots N-1)$.

Since the polynomials $r_{n}$ are orthogonal their roots are simple and real. For arbitrary real $C$, the polynomials $q_{n}$ have real coefficients. This means that any complex roots of $q_{n}$ come as half of a complex conjugate pair of roots. But as $C$ gets large the roots of $q_{n}$ approach the roots of the $r_{n}$ and it is impossible for two complex conjugate roots to approach two distinct roots of $r_{n}$. Thus for sufficiently large $C$ the roots of the first $N$ polynomials of $q_{n}$ are real and interleaved. Note that this interleaving is a strict interleaving so that no root of $q_{n}$ is equal to a root of $q_{n+1}$ for $0 \leq n<N-1$. Thus the roots of the first $N$ polynomials of $p$ are real and interleaved.

Now we let $C$ decrease until the interleaving property fails. It is not hard to see that the interleaving property can only fail if there is a transition value for $C$ and a $k$ with $0<k<N-1$ such that $q_{k}$ and $q_{k+1}$ have a common real root. Let that root be $x_{0}$. We will now demonstrate that such a transition point can only occur if $C$ is strictly less than 3 .

Consider the cubic equation

$$
t^{3}-x_{0} t^{2}+C t+1=0
$$

Let $t_{1}, t_{2}, t_{3}$ be the roots of this equation. The remainder of the proof hinges on whether this equation has a double root or triple root.

\section{The roots are distinct}

First suppose that equation (8) does not have a double root. In that case, we can find some $a_{1}, a_{2}, a_{3}$ such that

$$
q_{n}\left(x_{0}\right)=a_{1} t_{1}^{n+2}+a_{2} t_{2}^{n+2}+a_{3} t_{3}^{n+2} .
$$

Now we have $q_{-2}\left(x_{0}\right)=q_{-1}\left(x_{0}\right)=q_{k}\left(x_{0}\right)=q_{k+1}\left(x_{0}\right)$. This leads to the following equations:

$$
\begin{aligned}
a_{1}+a_{2}+a_{3} & =0 \\
a_{1} t_{1}+a_{2} t_{2}+a_{3} t_{3} & =0 \\
a_{1} t_{1}^{k+2}+a_{2} t_{2}^{k+2}+a_{3} t_{3}^{k+2} & =0 \\
a_{1} t_{1}^{k+3}+a_{2} t_{2}^{k+3}+a_{3} t_{3}^{k+3} & =0
\end{aligned}
$$

Note that the $a_{1}, a_{2}, a_{3}$ cannot be trivial because

$$
a_{1} t_{1}^{2}+a_{2} t_{2}^{2}+a_{3} t_{3}^{2}=1 \text {. }
$$

Thus the following determinants are zero:

$$
\begin{aligned}
& \left|\begin{array}{ccc}
1 & 1 & 1 \\
t_{1} & t_{2} & t_{3} \\
t_{1}^{k+2} & t_{2}^{k+2} & t_{3}^{k+2}
\end{array}\right|=0 \\
& \left|\begin{array}{ccc}
1 & 1 & 1 \\
t_{1} & t_{2} & t_{3} \\
t_{1}^{k+3} & t_{2}^{k+3} & t_{3}^{k+3}
\end{array}\right|=0
\end{aligned}
$$


This means in turn that we can find non-trivial $\alpha, \beta, \gamma$ and $\alpha^{\prime}, \beta^{\prime}, \gamma^{\prime}$ such that

$$
\begin{aligned}
\alpha+\beta t_{i}+\gamma t_{i}^{k+2} & =0 \\
\alpha^{\prime}+\beta^{\prime} t_{i}+\gamma^{\prime} t_{i}^{k+3} & =0
\end{aligned}
$$

for $i=1,2,3$. A little manipulation gives the following equations

$$
-\alpha^{\prime} \gamma+\left(\alpha \gamma^{\prime}-\beta^{\prime} \gamma\right) t_{i}+\beta \gamma^{\prime} t_{i}^{2}=0
$$

where $i=1,2,3$. The next question is whether equations (9) could be trivial in the sense that

$$
-\alpha^{\prime} \gamma=0, \quad\left(\alpha \gamma^{\prime}-\beta^{\prime} \gamma\right)=0, \quad \beta \gamma^{\prime}=0 .
$$

We will show that if equations (9) are trivial then $C<3$. This will be done in three cases. First, if $\gamma=0$ then $t_{i}=-\alpha / \beta$ and we find that there is a triple root which is a case that is covered later. Second, if $\gamma^{\prime}=0$ then $t_{i}=-\alpha^{\prime} / \beta^{\prime}$ which also leaves us in the triple root case. Finally, the only remaining case is that $\alpha^{\prime}=0$ and $\beta=0$. In this case,

$$
t_{i}^{k+2}=-\alpha / \gamma
$$

This means that the $t_{i}$ 's differ from one another by a factor of a root of unity. Also $1=|-1|=$ $\left|t_{1} t_{2} t_{3}\right|=\left|t_{1}\right|^{3}$ so $\left|t_{1}\right|=1$. But

$$
C=t_{1} t_{2}+t_{1} t_{3}+t_{2} t_{3}
$$

which means that $C<3$.

Thus the equations (9) are not trivial. But this means that the following determinant is zero:

$$
\left|\begin{array}{ccc}
1 & 1 & 1 \\
t_{1} & t_{2} & t_{3} \\
t_{1}^{2} & t_{2}^{2} & t_{3}^{2}
\end{array}\right|=\left(t_{3}-t_{2}\right)\left(t_{3}-t_{1}\right)\left(t_{2}-t_{1}\right)=0
$$

So there is a double root which was a case we are covering below.

\section{Double Root Case}

We will assume that the cubic equation (8) has a double root. Note that we are considering the triple root case to be distinct and it is handled below. If we have a double root then we can write

$$
t_{1}=t_{2}=-\phi, t_{3}=-\frac{1}{\phi^{2}}
$$

where $\phi \neq 1$. Note that $\phi$ must be real. Now we can find real numbers $\rho, \sigma, \tau$ such that

$$
q_{n-2}\left(x_{0}\right)=(\rho n+\sigma)(-\phi)^{n}+\tau\left(-1 / \phi^{2}\right)^{n}
$$

Using $q_{-2}\left(x_{0}\right)=q_{-1}\left(x_{0}\right)=0$, we can solve for $\rho, \sigma$ and $\tau$ to get

$$
q_{n-2}\left(x_{0}\right)=\sigma(-\phi)^{n}\left[\left(\frac{1}{\phi^{3}}-1\right) n+1-\frac{1}{\phi^{3 n}}\right]
$$

THE ELECTRONiC JOURNAL OF COMBINATORICS 8 (2001), \#R36 
Now we will use the claim that $q_{k}\left(x_{0}\right)=0$ for $k>0$. In this case, we would have

$$
k+2=\frac{1-(1 / \phi)^{3 k+6}}{1-(1 / \phi)^{3}}=1+\frac{1}{\phi^{3}}+\ldots+\frac{1}{\phi^{3 k+3}} .
$$

Note that the right hand side of this equation has $k+2$ summands. If $\phi>0$ then we look at the cases where $\phi>1$ and $\phi<1$. In both cases the above equality is impossible. If $\phi<0$ then we use the fact that

$$
C=\phi^{2}+\frac{2}{\phi} .
$$

For negative $\phi$ the right hand side of this equation is decreasing with $\phi$. It ranges from $+\infty$ as $\phi \rightarrow-\infty$ to $-\infty$ as $\phi \rightarrow 0-$. Thus $C$ can only be greater than or equal to 3 if $\phi \leq-2$. But if $\phi \leq-2$ then the equation

$$
k+2=1+\frac{1}{\phi^{3}}+\ldots+\frac{1}{\phi^{3 k+3}}
$$

is clearly impossible.

\section{Triple Root Case}

We are left with only one possible remaining case: that of triple roots. In that case $t_{1}=t_{2}=$ $t_{3}=-1, C=3$ and $x_{0}=-3$. We then have

$$
q_{n+1}\left(x_{0}\right)=-3 q_{n}\left(x_{0}\right)-3 q_{n-1}\left(x_{0}\right)-q_{n-2}\left(x_{0}\right)
$$

and

$$
q_{n}\left(x_{0}\right)=\frac{(n+1)(n+2)}{2} .
$$

This covers all the cases. It means that the transition point that we have been talking about cannot happen for $C \geq 3$. Thus if $C \geq 3$ the roots of the $q_{n}$ are real and interleaved.

\section{The 6-Conjecture}

All of the work to this point derives from the initial consideration of the $T$-transform of the powers $(x(x-1)+r)^{n}$ and the 4 -term polynomial sequences they satisfy. Of course we could begin with the powers of other polynomials invariant under $x \rightarrow 1-x$, and consider the higher order sequences they define. We then would consider which values of various parameters guarantee a Riemann hypothesis.

For the sake of brevity, we look at just one more case of the kind of situation that presents itself in section 11, and skip the derivations.

We have chosen a 5 -term sequence, $q_{n}=q_{n}(x, C)$ with

$$
q_{n}=x q_{n-1}-C q_{n-2}+4 q_{n-3}-q_{n-4}
$$


with initial terms

$$
\begin{array}{r}
q_{-3}=0 \\
q_{-2}=0 \\
q_{-1}=0 \\
q_{0}=1
\end{array}
$$

as an example of an infinite class of sequences depending on a single parameter $C$ and we begin with the following conjecture.

Conjecture 2 (The 6-Conjecture) The sequence of polynomials satisfying the recursion

$$
q_{n}=x q_{n-1}-C q_{n-2}+4 q_{n-3}-q_{n-4}
$$

with initial polynomials as in (11) have real zeros if and only if $C \geq 6$. In this case, the zeros of $q_{n+1}$ interlace the zeros of $q_{n}$.

Numerical evidence indicates that many other polynomial sequences depending on a single parameter $C$ have real zeros if and only if $C$ is not smaller than some critical value. We connect these higher order sequences to Hankel determinants in section 11. There is a substantial amount of numerical evidence that the critical coefficients that are at work for these recursions come from binomial coefficients, e. g. 3,1 for 4-term recursions, and 6,4,1 for 5-term recursions.

\section{Moments}

We consider the sequence of polynomials $q_{n}=q_{n}(x)$ defined by the 4-term recursion

$$
q_{n}=x q_{n-1}-C q_{n-2}-q_{n-3}, \quad(n \geq 1)
$$

with $q_{-2}=q_{-1}=0$, and $q_{0}=1$. Thus

$$
\begin{aligned}
& q_{0}=1 \\
& q_{1}=x \\
& q_{2}=x^{2}-C \\
& q_{3}=x^{3}-2 C x-1 \\
& q_{4}=x^{4}-3 C x^{2}-2 x+C^{2}
\end{aligned}
$$

Write

$$
q_{n}(x)=\sum_{j=0}^{n} d_{n, j} x^{j}
$$

and define $\mathcal{Q}_{n}=\left[d_{i, j}\right]_{0 \leq i, j \leq n}$ to be the $(n+1) \times(n+1)$ matrix of coefficients. Thus

$$
\mathcal{Q}_{4}=\left[\begin{array}{ccccc}
1 & 0 & 0 & 0 & 0 \\
0 & 1 & 0 & 0 & 0 \\
-C & 0 & 1 & 0 & 0 \\
-1 & -2 C & 0 & 1 & 0 \\
C^{2} & -2 & -3 C & 0 & 1
\end{array}\right]
$$

THE ELECTRONiC JOURNAL OF COMBINATORICS 8 (2001), \#R36 
We specify a linear functional $\mathcal{L}_{C}$ on the space of real polynomials by

$$
\begin{aligned}
\mathcal{L}_{C}\left[q_{0}\right] & =1 \\
\mathcal{L}_{C}\left[q_{n}\right] & =0, \quad n \geq 1
\end{aligned}
$$

Expressing the moments of $\mathcal{L}_{C}$ by

$$
\mu_{n}=\mu_{n}(C)=\mathcal{L}_{C}\left[x^{n}\right]
$$

then the first few moments are

$$
\begin{aligned}
& \mu_{0}=1 \\
& \mu_{1}=0 \\
& \mu_{2}=C \\
& \mu_{3}=1 \\
& \mu_{4}=2 C^{2} \\
& \mu_{5}=5 C \\
& \mu_{6}=3+5 C^{3},
\end{aligned}
$$

and in general, we have the following result.

Theorem 2 The moments $\mu_{n}$ of the functional $\mathcal{L}$ are given by any of the following expressions:

1. The $(n, 0)$-th entry of $\mathcal{Q}_{n}^{-1}$.

2. The sum of the weights of all lattice paths from the origin to the point $(n, 0)$ with elementary steps

$$
\begin{aligned}
& (a, b) \rightarrow(a+1, b+1) \quad \text { with weight } 1, \\
& (a, b) \rightarrow(a+1, b-1) \quad \text { with weight } C, \\
& (a, b) \rightarrow(a+1, b-2) \quad \text { with weight } 1,
\end{aligned}
$$

which stay weakly above the $x$-axis.

3. The sum of the monomials $C^{n_{2}(T)}$ over all 2-3-trees $T$ on $n+1$ nodes, where $n_{2}(T)=$ number of nodes of $T$ with 2 children.

4. The coefficient of $x^{n}$ in

$$
\frac{1}{n+1}\left(1+C x^{2}+x^{3}\right)^{n+1}
$$

5. The sum

$$
\frac{1}{n+1} \sum_{n=3 j+2 k}\left(\begin{array}{c}
n+1 \\
j, k
\end{array}\right) C^{k}
$$

THE ELECTRONIC JOURNAL OF COMBINATORICS 8 (2001), \#R36 
6. For $C \geq 3$, the integral moment

$$
\int_{t_{2}}^{t_{1}} t^{n} w(t) d t
$$

where $t_{2}<t_{1}$ are the two larger roots of the discriminant of $z^{3}+C z^{2}-t z+1$, and $w(t)=w_{C}(t)$ is positive for $t_{2}<t<t_{1}$.

7. For $C=3$, the integral moment

$$
\frac{\sqrt{3}}{2 \pi} 3^{n+4} \int_{0}^{1} f^{n}(u) g(u) d u
$$

where

$$
\begin{aligned}
& f(u)=9 u(1-u)-1 \\
& g(u)=u^{\frac{1}{3}}(1-u)^{\frac{2}{3}}(1-2 u)
\end{aligned}
$$

8. For $C=3$, the expression

$$
(-1)^{n} 3^{n+4} \sum_{k=0}^{2 n+1} c_{n, k}\left(\begin{array}{c}
k+\frac{1}{3} \\
2 n+3
\end{array}\right)
$$

where the $c_{n, k}$ are defined by

$$
(u+1)\left(1+7 u+u^{2}\right)^{n}=\sum_{k=0}^{2 n+1} c_{n, k} u^{k} .
$$

9. For $C=3$, the expression

$$
(-1)^{n} 3^{n+4} \sum_{k=0}^{n}\left(\begin{array}{l}
n \\
k
\end{array}\right)\left(\begin{array}{c}
k+\frac{1}{3} \\
2 k+3
\end{array}\right) \frac{3^{2 k}}{3 k+5} .
$$

Proof To prove part 1, note that by (12) and (13), $\mu_{0}=1$ and for $i>0$

$$
\sum_{j=0}^{i} d_{i, j} \mu_{j}=0 .
$$

Therefore for every $n>0$,

$$
\mathcal{Q}_{n}\left[\begin{array}{c}
\mu_{0} \\
\mu_{1} \\
\vdots \\
\mu_{n}
\end{array}\right]=\left[\begin{array}{c}
1 \\
0 \\
\vdots \\
0
\end{array}\right]
$$

Thus the vector $\left[\mu_{0}, \mu_{1}, \cdots, \mu_{n}\right]^{t}$ is the first column of $\mathcal{Q}_{n}^{-1}$ and (1) follows.

To prove 2 , let $\mathcal{Q}_{n}^{-1}=\left[e_{i, j}\right]_{0 \leq i, j \leq n}$. Thus

$$
x^{i}=\sum_{j=0}^{n} e_{i, j} q_{j}(x)
$$

THE ELECTRONIC JOURNAL OF COMBINATORICS 8 (2001), \#R36 
Multiplying both sides by $x$,

$$
\begin{aligned}
x^{i+1} & =\sum_{j=0}^{n} e_{i, j} x q_{j}(x) \\
& =\sum_{j=0}^{n} e_{i, j}\left(q_{j+1}(x)+C q_{j-1}(x)+q_{j-2}(x)\right) \\
& =q_{i+1}(x)+C e_{i, 1}+e_{i, 2}+\sum_{j=1}^{n} e_{i, j-1} q_{j}(x)+\sum_{j=1}^{n} C e_{i, j+1} q_{j}(x)+\sum_{j=1}^{n} e_{i, j+2} q_{j}(x)
\end{aligned}
$$

Comparing coefficients with the expansion (18) with $i$ replaced by $i+1$

$$
e_{i+1, j}= \begin{cases}1 & \text { if } j=i+1 \\ e_{i, j-1}+C e_{i, j+1}+e_{i, j+2} & \text { if } 0<j \leq i \\ C e_{i, 1}+e_{i, 2} & \text { if } j=0\end{cases}
$$

This is the same recursion satisfied by the sum of the weights of the collection of paths from

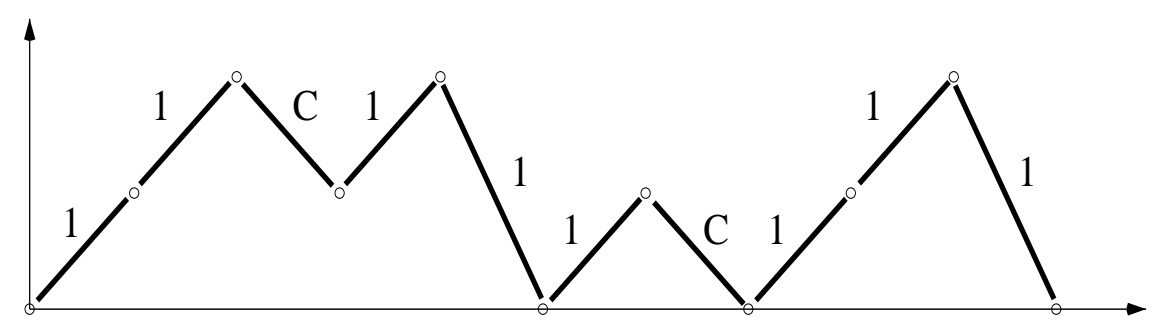

Figure 2: A lattice path from the origin to (10,0) with elementary steps as in (14).

the origin to the point $(i+1, j)$ which stay weakly above the $x$-axis and have elementary steps given in (14). An example of such a path from the origin to $(10,0)$ with weight $C^{2}$ is shown in Figure 2. Since the value at the lattice point $(n, 0)$ is $e_{n, 0}$, the sum of the weights of all paths from the origin to $(n, 0)$ is $\mu_{n}$ by part 1 . This proves part 2 .

To prove part 3 , we traverse a lattice path in part 2 from right to left, coding the three elementary steps in (14) by $x_{0}, x_{2}$, and $x_{3}$, respectively, and padding the resulting string with an extra $x_{0}$. For the example path in Figure 2 this results in the code

$$
x_{3} x_{0} x_{0} x_{2} x_{0} x_{3} x_{0} x_{2} x_{0} x_{0} x_{0}
$$

This word is the word obtained by the depth-first traversal of a 2-3-tree $T$ on 11 nodes, and putting the labels of the nodes down one by one from left to right. Each $x_{3}$ is the label of an internal node with 3 children, each $x_{2}$ is the label of an internal node with 2 children, and $x_{0}$ 's are the labels of leaf nodes with no children (thus the internal nodes have 2 or 3 children, as suggested by the name 2-3-tree). Note that $n_{0}+n_{2}+n_{3}=n+1$ where $n_{i}$ is the number of nodes with $i$ children, and the contribution of the tree is $C^{n_{2}(T)}$, since under this bijection, the 


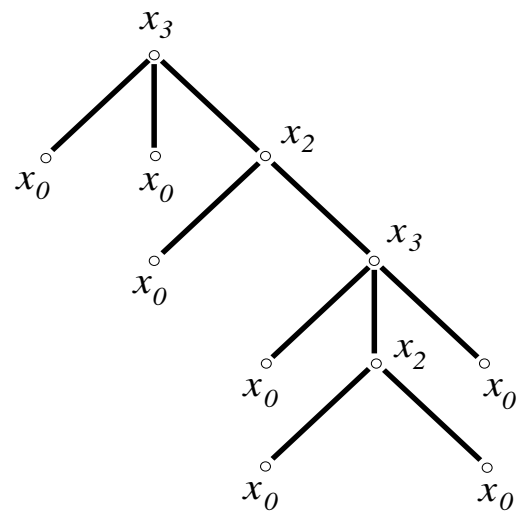

Figure 3: The 2-3-tree corresponding to the lattice path in Figure 2.

nodes labeled with $x_{2}$ have weight $C$, and all the other nodes have weight 1 . The tree that corresponds to the path in Figure 2 via the depth-first code in (20) is shown in Figure 3.

To prove part 4, we use the following version of Lagrange Inversion

Theorem (Lagrange Inversion Formula) Let $R(x)$ be the formal power series

$$
R(x)=R_{0}+R_{1} x+R_{2} x^{2}+\cdots
$$

and let

$$
f(x)=f_{1} x+f_{2} x^{2}+f_{3} x^{3}+\cdots
$$

be the formal power series solution of the equation $f(x)=x R(f(x))$. Then $f_{n}$ is given by the coefficient of $x^{n-1}$ in $\frac{1}{n} R^{n}(x)$.

We use this result in the following way. Let

$$
f(x)=\sum_{T} C^{n_{2}(T)} x^{n(T)}=\sum_{n \geq 0} \mu_{n}(C) x^{n+1}
$$

where the sum is over all 2-3-trees $T$, and $n(T)$ is the total number of nodes in $T$. Any 2-3-tree with more than one node can be uniquely decomposed into either 2 or 3 principal subtrees. Therefore $f(x)$ satisfies the functional equation

$$
f(x)=x+x C f(x)^{2}+x f(x)^{3}
$$

Now we can use the Lagrange Inversion Formula with $R(x)=1+C x^{2}+x^{3}$ and obtain $\mu_{n}=f_{n+1}$ as the coefficient of $x^{n}$ in $\frac{1}{n+1}\left(1+C x^{2}+x^{3}\right)^{n+1}$. This proves part 4 . Part 5 follows by the multinomial theorem.

Parts 4 and 5 of the theorem have alternate proofs. We begin with the series

$$
\sum_{0}^{\infty} z^{k} q_{k}(x),
$$

THE ELECTRONiC JOURNAL OF COMBINATORICS 8 (2001), \#R36 
which may be evaluated via the recursion

$$
\begin{aligned}
\sum_{0}^{\infty} z^{k} q_{k}(x) & =1+\sum_{1}^{\infty} z^{k}\left(x q_{k-1}-C q_{k-2}-q_{k-3}\right) \\
& =1+\left(z x-C z^{2}-z^{3}\right) \sum_{0}^{\infty} z^{k} q_{k}
\end{aligned}
$$

to obtain

$$
\sum_{0}^{\infty} z^{k} q_{k}(x)=\frac{1}{z(t(z)-x)}
$$

where

$$
t(z)=\frac{1}{z}+C z+z^{2}
$$

Let $\mathcal{T}(\epsilon)$ denote the image of the circle $[z:|z|=\epsilon]$ under the map $z \rightarrow t(z)$. Given $C$, if $\epsilon$ is sufficiently small, as $z$ goes around the circle $z=\epsilon, t(z)$ goes around the origin once in the opposite direction. It follows that

$$
\begin{aligned}
x^{n} & =\frac{1}{2 \pi i} \oint_{\mathcal{T}(\epsilon)} \frac{t^{n}}{t-x} d t \\
& =-\frac{1}{2 \pi i} \oint_{|z|=\epsilon} \frac{t^{\prime}(z) t^{n}(z)}{t-x} d z \\
& =\sum_{k=0}^{\infty}\left(-\frac{1}{2 \pi i} \oint_{|z|=\epsilon} t^{\prime} t^{n} z^{k+1} d z\right) q_{k}(x) .
\end{aligned}
$$

This sum is finite, and we therefore obtain

$$
\mathcal{L}\left[x^{n}\right]=-\frac{1}{2 \pi i} \oint_{|z|=\epsilon} t^{\prime} t^{n} z d z
$$

as $\mathcal{L}$ simply picks off the $k=0$ term in the sum. It follows that

$$
\begin{aligned}
\mathcal{L}\left[x^{n}\right] & =\frac{1}{2(n+1) \pi i} \oint_{|z|=\epsilon} t^{n+1} d z \\
& =\frac{1}{2(n+1) \pi i} \oint_{|z|=\epsilon}\left(1+C z^{2}+z^{3}\right)^{n+1} \frac{d z}{z^{n+1}} \\
& =\frac{1}{n+1} \sum_{n=3 j+2 k}\left(\begin{array}{c}
n+1 \\
j, k
\end{array}\right) C^{k} .
\end{aligned}
$$

This again establishes parts 4 and 5 of the theorem.

For the proof of the parts 6 and 7, we convert the path integral defining $\mu_{n}$ in (21) to a real integral on the real line. We begin with the assumption that $C>3$. We denote

$$
\begin{aligned}
p(z) & =z^{3}+C z^{2}-t z+1 \\
& =\left(z-z_{1}(t)\right)\left(z-z_{2}(t)\right)\left(z-z_{3}(t)\right) .
\end{aligned}
$$


Then

$$
\begin{aligned}
& z_{1}(t)=-\frac{C}{3}-\frac{1}{2^{\frac{1}{3}}}\left(H_{1}^{\frac{1}{3}}+H_{2}^{\frac{1}{3}}\right) \\
& z_{2}(t)=-\frac{C}{3}-\frac{1}{2^{\frac{1}{3}}}\left(\omega^{2} H_{1}^{\frac{1}{3}}+\omega H_{2}^{\frac{1}{3}}\right) \\
& z_{3}(t)=-\frac{C}{3}-\frac{1}{2^{\frac{1}{3}}}\left(\omega H_{1}^{\frac{1}{3}}+\omega^{2} H_{2}^{\frac{1}{3}}\right)
\end{aligned}
$$

where $H_{1}, H_{2}$, and the discriminant $\Delta$ of $p(z)$ are given as

$$
\begin{aligned}
w(t) & =\frac{\sqrt{3}}{2 \pi 2^{\frac{1}{3}}}\left(H_{2}^{\frac{1}{3}}-H_{1}^{\frac{1}{3}}\right) \\
H_{1} & =G+\sqrt{-\frac{\Delta}{27}} \\
H_{2} & =G-\sqrt{-\frac{\Delta}{27}} \\
G & =1+\frac{t C}{3}+2\left(\frac{C}{3}\right)^{3} \\
-\frac{\Delta}{27} & =1+\frac{4 C^{3}}{27}+\frac{2 C t}{3}-\frac{C^{2} t^{2}}{27}-\frac{4 t^{3}}{27} .
\end{aligned}
$$

Since $C>3$ by assumption, the discriminant of $p$ has three distinct real $t$-roots $t_{1}(C), t_{2}(C)$, and $t_{3}(C)$, satisfying

$$
t_{3}(C)<-C<t_{2}(C)<0
$$

and

$$
\frac{15}{4}<t_{1}(C)<C+2
$$

We let $z_{1}$ be the real branch of $p(z)=0$, and we observe that $t_{1}(C), t_{2}(C)$, and $t_{3}(C)$ are each 2-cycles of the branches, $z_{1}(t), z_{2}(t)$, and $z_{3}(t)$, where

$$
\begin{aligned}
0<z_{2}\left(t_{1}\right)=z_{3}\left(t_{1}\right) & <\frac{1}{2} \\
z_{1}\left(t_{1}\right) & <-4
\end{aligned}
$$

and

$$
\begin{aligned}
-1<z_{2}\left(t_{2}\right)= & z_{3}\left(t_{2}\right)<0 \\
z_{1}\left(t_{2}\right) & <-1
\end{aligned}
$$

and

$$
\begin{aligned}
z_{1}\left(t_{3}\right)=z_{2}\left(t_{3}\right) & <-1 \\
-1<z_{3}\left(t_{3}\right) & <0 .
\end{aligned}
$$

Next, note that if $\mathcal{T}=\mathcal{T}(\mathcal{C})$ denotes the image of the unit circle $|z|=1$ under the map

$$
z \rightarrow t(z)
$$


then $\mathcal{T}$ traverses the origin in the $t$-plane once, cutting the real axis at $-C$ and $C+2$. By the inequalities above, the two roots $t_{2}<t_{1}$ therefore lie within this contour, while the third root $t_{3}$ is outside. See Figure 4.
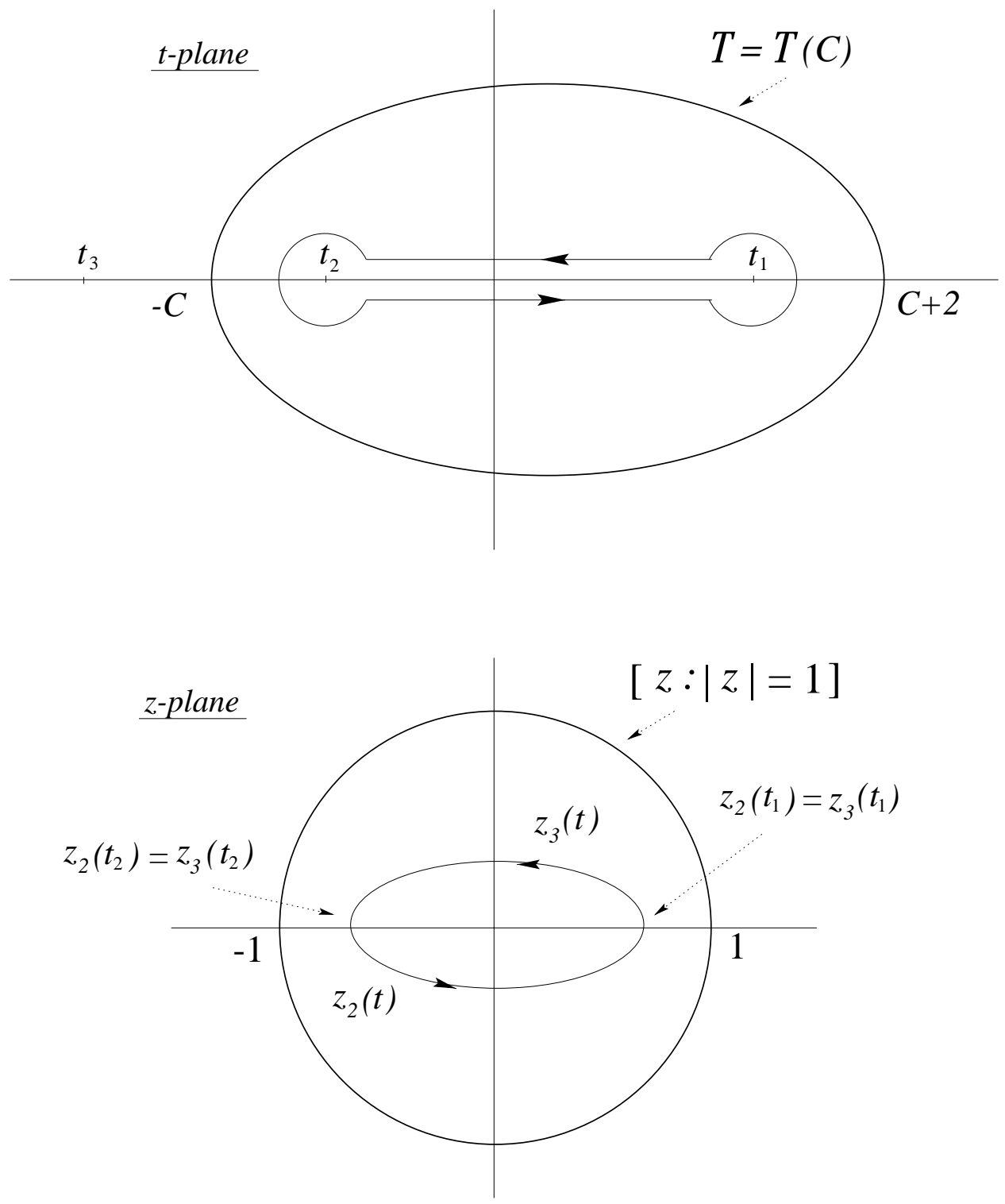

Figure 4: Paths of integration in the $z$ and the $t$-planes.

Since

$$
\begin{aligned}
\mathcal{L}\left[x^{n}\right] & =-\frac{1}{2 \pi i} \oint_{|z|=\epsilon} t^{\prime} t^{n} z d z \\
& =-\frac{1}{2 \pi i} \oint_{|z|=1} t^{\prime} t^{n} z d z
\end{aligned}
$$


we can convert this $z$-integral to an integral in the $t$-plane,

$$
\begin{aligned}
\mu_{n} & =\frac{1}{2 \pi i} \oint_{\mathcal{T}} z t^{n} d t \\
& =\frac{1}{2 \pi i} \int_{t_{2}}^{t_{1}}\left(z_{2}(t)-z_{3}(t)\right) t^{n} d t \\
& =\frac{\omega-\omega^{2}}{2 \pi i 2^{\frac{1}{3}}} \int_{t_{2}}^{t_{1}}\left(H_{1}^{\frac{1}{3}}(t)-H_{2}^{\frac{1}{3}}(t)\right) t^{n} d t \\
& =\frac{\sqrt{3}}{2 \pi 2^{\frac{1}{3}}} \int_{t_{2}}^{t_{1}}\left(H_{1}^{\frac{1}{3}}(t)-H_{2}^{\frac{1}{3}}(t)\right) t^{n} d t \\
& =\int_{t_{2}}^{t_{1}} \omega(t) t^{n} d t
\end{aligned}
$$

which is part 6 of the theorem.

At $C=3$, the branching of the function $z(t)$ changes a bit, because the discriminant

$$
\Delta=-(t+3)^{2}(15-4 t)
$$

now has a double root at -3 . Hence, $t_{2}(3)=-3$ is a 3 -cycle (and $t_{1}(3)=\frac{15}{4}$ remains a 2cycle). But this fact clearly does not change the argument above and we simply take the limit as $C \rightarrow 3^{+}$, to obtain

$$
=\frac{\sqrt{3}}{2 \pi 2^{\frac{1}{3}}} \int_{-3}^{\frac{15}{4}}\left(H_{1}^{\frac{1}{3}}(t)-H_{2}^{\frac{1}{3}}(t)\right) t^{n} d t
$$

We use this last expression to obtain the formula of part 7 . Since $C=3$, we have

$$
-\frac{\Delta}{27}=\frac{\left.(t+3)^{2}(15-4 t)\right)}{27}
$$

in which case we have

$$
\begin{aligned}
& H_{1}(t)=(3+t)\left[1+\frac{\sqrt{15-4 t}}{3 \sqrt{3}}\right] \\
& H_{2}(t)=(3+t)\left[1-\frac{\sqrt{15-4 t}}{3 \sqrt{3}}\right]
\end{aligned}
$$

Making the change of variable,

$$
3+t=\frac{27}{4} u \quad d t=\frac{27}{4} d u
$$

in the integral defining $\mathcal{L}\left[x^{n}\right]$ we get

$$
\begin{aligned}
\mu_{n} & =\frac{\sqrt{3}}{2 \pi 2^{\frac{1}{3}}} \int_{0}^{1}\left(\frac{27}{4} u\right)^{\frac{1}{3}}\left[(1+\sqrt{1-u})^{\frac{1}{3}}-(1-\sqrt{1-u})^{\frac{1}{3}}\right]\left(\frac{27}{4} u-3\right)^{n} \frac{27}{4} d u \\
& =3^{n+4} \frac{\sqrt{3}}{16 \pi} \int_{0}^{1} u^{\frac{1}{3}}\left[(1+\sqrt{1-u})^{\frac{1}{3}}-(1-\sqrt{1-u})^{\frac{1}{3}}\right]\left(\frac{9}{4} u-1\right)^{n} d u .
\end{aligned}
$$

Next make the change of variable

$$
\begin{aligned}
u & =4 v(1-v) \\
d u & =4(1-2 v) d v
\end{aligned}
$$


to obtain

$$
\begin{aligned}
\mu_{n} & =3^{n+4} \frac{\sqrt{3}}{2 \pi} \int_{0}^{\frac{1}{2}} f^{n}(v)[v(1-v)]^{\frac{1}{3}}\left[(1-v)^{\frac{1}{3}}-v^{\frac{1}{3}}\right](1-2 v) d v \\
& =3^{n+4} \frac{\sqrt{3}}{2 \pi} \int_{0}^{1} f^{n}(v) g(v) d v
\end{aligned}
$$

where $f$ and $g$ are as in (16).

To prove part 8 , we begin with the expression,

$$
\mu_{n}=3^{n+4} \frac{\sqrt{3}}{16 \pi} \int_{0}^{1} u^{\frac{1}{3}}\left[(1+\sqrt{1-u})^{\frac{1}{3}}-(1-\sqrt{1-u})^{\frac{1}{3}}\right]\left(\frac{9}{4} u-1\right)^{n} d u .
$$

The substitution

$$
\begin{aligned}
u & =1-v^{2} \\
d u & =-2 v d v
\end{aligned}
$$

results in the difference of two integrals

$$
\mu_{n}=3^{n+4} \frac{\sqrt{3}}{16 \pi} \int_{0}^{1}\left(1-v^{2}\right)^{\frac{1}{3}}\left[(1+v)^{\frac{1}{3}}-(1-v)^{\frac{1}{3}}\right]\left(\frac{9}{4}\left(1-v^{2}\right)-1\right)^{n} 2 v d v .
$$

In the second integral, let $v=-w, d v=-d w$, and combine the result with the first integral to get

$$
\mu_{n}=3^{n+4} \frac{\sqrt{3}}{8 \pi} \int_{-1}^{1}\left(1-v^{2}\right)^{\frac{1}{3}}\left[(1+v)^{\frac{1}{3}}\right]\left(\frac{9}{4}\left(1-v^{2}\right)-1\right)^{n} v d v .
$$

Then make the substitution

$$
\begin{aligned}
v & =\frac{1-u}{1+u} \\
d v & =\frac{-2}{(1+u)^{2}} d u
\end{aligned}
$$

and note that

$$
\begin{aligned}
& 1-v=\frac{2 u}{1+u} \\
& 1+v=\frac{2}{1+u}
\end{aligned}
$$

to obtain

$$
\begin{aligned}
\mu_{n} & =3^{n+4} \frac{\sqrt{3}}{2 \pi} \int_{0}^{\infty} u^{\frac{1}{3}} \frac{1-u}{(1+u)^{4}}\left[\left(\frac{3}{1+u}\right)^{2} u-1\right]^{n} d u \\
& =(-1)^{n} 3^{n+4} \frac{\sqrt{3}}{2 \pi} \int_{0}^{\infty} u^{\frac{1}{3}} \frac{1-u}{(1+u)^{2 n+4}}\left[1-7 u+u^{2}\right]^{n} d u \\
& =(-1)^{n} 3^{n+4} \frac{\sqrt{3}}{2 \pi} \int_{0}^{\infty} \frac{u^{\frac{1}{3}}}{(1+u)^{2 n+4}} \sum_{k=0}^{2 n+1}(-1)^{k} c_{n, k} u^{k} d u
\end{aligned}
$$


With $0<a<m+1$, we have

$$
\int_{0}^{\infty} \frac{u^{a-1}}{(1+u)^{m+1}} d u=(-1)^{m} \frac{\pi}{\sin (a \pi)}\left(\begin{array}{c}
a-1 \\
m
\end{array}\right)
$$

which readily provides the expression in part 8 for $\mu_{n}$. Part 9 of the theorem follows from an alternate evaluation of the integral formula (22). We omit the details.

\section{Remark}

Part 8 of the above provides new expressions for the moments $\mu_{n}$ in terms of sums of fractional binomial coefficients. Thus, if $n=0$, we have

$$
\mu_{0}=3^{4}\left[\left(\begin{array}{l}
\frac{1}{3} \\
3
\end{array}\right)+\left(\begin{array}{l}
\frac{4}{3} \\
3
\end{array}\right)\right]=1
$$

Similarly

$$
\mu_{1}=(-1) 3^{5}\left[\left(\begin{array}{l}
\frac{1}{3} \\
5
\end{array}\right)+8\left(\begin{array}{l}
\frac{4}{3} \\
5
\end{array}\right)+8\left(\begin{array}{l}
\frac{7}{3} \\
5
\end{array}\right)+\left(\begin{array}{c}
\frac{10}{3} \\
5
\end{array}\right)\right]=0
$$

and

$$
\mu_{2}=3^{6}\left[\left(\begin{array}{l}
\frac{1}{3} \\
7
\end{array}\right)+15\left(\begin{array}{l}
\frac{4}{3} \\
7
\end{array}\right)+65\left(\begin{array}{l}
\frac{7}{3} \\
7
\end{array}\right)+65\left(\begin{array}{c}
\frac{10}{3} \\
7
\end{array}\right)+15\left(\begin{array}{c}
\frac{13}{3} \\
7
\end{array}\right)+\left(\begin{array}{c}
\frac{16}{3} \\
7
\end{array}\right)\right]=3 .
$$

\section{Remark}

It is evident from the lattice path interpretation in part 2, and multinomial expansion in part 5 of Theorem 2 that as a polynomial in $C$,

$$
\operatorname{deg}\left(\mu_{2 n}(C)\right)=n, \quad \operatorname{deg}\left(\mu_{2 n+1}(C)\right)=n-1 .
$$

Furthermore the coefficient of the leading term in $\mu_{n}(C)$ is the Catalan number $\frac{1}{2 n+1}\left(\begin{array}{c}2 n+1 \\ n\end{array}\right)$ for $\mu_{2 n}(n>0)$, and the binomial coefficient $\left(\begin{array}{c}2 n+1 \\ n+1\end{array}\right)$ for $\mu_{2 n+1}$.

\section{$5 \quad$ Very Special Hankel Determinants}

Consider again the sequence of polynomials defined by the 4-term recursion

$$
q_{n}=x q_{n-1}-C q_{n-2}-q_{n-3}, \quad(n \geq 1)
$$

with $q_{-2}=q_{-1}=0$, and $q_{0}=1$, and the linear functional $\mathcal{L}_{C}$ defined by

$$
\begin{gathered}
\mathcal{L}_{C}\left[q_{0}\right]=1, \quad \mathcal{L}_{C}\left[q_{n}\right]=0 \quad(n \geq 1) . \\
\mu_{n}=\mu_{n}(C)=\mathcal{L}_{C}\left[x^{n}\right]
\end{gathered}
$$


as characterized by Theorem 2 . For the critical value $C=3$, we have

$$
\begin{aligned}
& \mu_{0}=1 \\
& \mu_{1}=0 \\
& \mu_{2}=3 \\
& \mu_{3}=1 \\
& \mu_{4}=18 \\
& \mu_{5}=15,
\end{aligned}
$$

and these in turn produce a sequence of Hankel determinants as defined in (7) that start out as

$$
\begin{aligned}
& \Delta_{0}=1 \\
& \Delta_{1}=3 \\
& \Delta_{2}=26 \\
& \Delta_{3}=646 \\
& \Delta_{4}=45885 \\
& \Delta_{5}=930465
\end{aligned}
$$

and continue to agree (as far as the tables go) with the number of ASMs with vertical symmetry given by the formula (34) for $R R(n)$, and so you are suddenly working in another universe. Since the critical value is $C=3$, write $C=3+t$ with $t \geq 0$ and let $\mu_{n}=\mu_{n}(3+t)$. We find that

$$
\begin{aligned}
& \mu_{0}=1 \\
& \mu_{1}=0 \\
& \mu_{2}=3+t \\
& \mu_{3}=1 \\
& \mu_{4}=18+12 t+2 t^{2} \\
& \mu_{5}=15+5 t \\
& \mu_{6}=138+135 t+45 t^{2}+5 t^{3} \\
& \mu_{7}=189+126 t+21 t^{2} \\
& \mu_{8}=1218+1540 t+756 t^{2}+168 t^{3}+14 t^{4} \\
& \mu_{9}=2280+2268 t+756 t^{2}+84 t^{3}
\end{aligned}
$$

As a consequence of parts 2 and 5 of Theorem 2, the $\mu_{n}(C)$ are polynomials in $C$ with nonnegative integral coefficients. It follows from (23) that also as polynomials in $t, \operatorname{deg}\left(\mu_{2 n}(3+t)\right)=$ $n, \operatorname{deg}\left(\mu_{2 n+1}(3+t)\right)=n-1$, and the coefficients of $\mu_{n}(3+t)$ are non-negative integers.

As polynomials in $C$, the first few Hankel determinants $\Delta_{n}=\operatorname{det}\left[\mu_{i+j}\right]$ are as shown below. Evidently, $\operatorname{deg}\left(\Delta_{n}(C)\right)=\frac{1}{2} n(n+1)$. However the coefficients of $\Delta_{n}(C)$ are not non-negative.

$$
\Delta_{0}=1
$$




$$
\begin{aligned}
& \Delta_{1}=C \\
& \Delta_{2}=-1+C^{3} \\
& \Delta_{3}=-2-3 C^{3}+C^{6} \\
& \Delta_{4}=-14 C-6 C^{7}+C^{10} \\
& \Delta_{5}=18-120 C^{3}-30 C^{6}+15 C^{9}-10 C^{12}+C^{15}
\end{aligned}
$$

But replacing $C$ by $3+t$, we obtain the polynomials $\Delta_{n}=\Delta_{n}(3+t)=\operatorname{det}\left[\mu_{i+j}(3+t)\right]$ as

$$
\begin{aligned}
\Delta_{0}= & 1 \\
\Delta_{1} & =3+t \\
\Delta_{2}= & 26+27 t+9 t^{2}+t^{3} \\
\Delta_{3}= & 646+1377 t+1188 t^{2}+537 t^{3}+135 t^{4}+18 t^{5}+t^{6} \\
\Delta_{4}= & 45885+166198 t+264627 t^{2}+245430 t^{3}+147420 t^{4}+ \\
& 60102 t^{5}+16884 t^{6}+3234 t^{7}+405 t^{8}+30 t^{9}+t^{10}
\end{aligned}
$$

It is interesting that these $\Delta_{n}(3+t)$ do have non-negative coefficients. We wonder whether or not this is true in general. The constant terms $1,3,26,646,45885, \ldots$ of $\Delta_{n}(3+t)$ agree with the number $V_{n}$ of ASMs with vertical symmetry as far as the tables go, as noted earlier. Furthermore, it is reasonable to think that ASMs with vertical symmetry are only a special set of objects enumerated by $\Delta_{n}(3+t)$, the others having some non-zero statistic indicated by the exponents of $t$.

\section{Positivity is Insufficient}

Given the analogy with 3 -term recursions, it is natural to conjecture that if the linear form $\mathcal{L}$ associated with a 4-term recursion is positive then the zeros of the recursively defined polynomials are real and interleaved. In this section we include an argument that shows that this conjecture is false.

We start with the positive linear form and then generate the badly behaved 4-term recursion to fit the linear form. We will define the positive linear form by starting with the set of orthogonal polynomials associated with the positive linear form. We can actually choose any set of orthogonal polynomials, but for completeness we choose the Hermite polynomials. The recursion for the Hermite polynomials $H_{n}=H_{n}(x)$ is as follows:

$$
\begin{aligned}
H_{-2} & =H_{-1}=0 \\
H_{n} & =2 x H_{n-1}-2(n-1) H_{n-2}
\end{aligned}
$$

The positive linear form then satisfies the equations

$$
\begin{aligned}
& \mathcal{L}\left[H_{0}\right]=1 \\
& \mathcal{L}\left[H_{n}\right]=0 \quad(n>0) .
\end{aligned}
$$

THE ELECTRONiC JOURNAL OF COMBINATORICS 8 (2001), \#R36 
Now we define the polynomials $q_{n}=q_{n}(x)$ that should satisfy the 4-term recursion as

$$
\begin{array}{lll}
q_{0} & =H_{0}=1 & \\
q_{1} & =H_{1} & \\
q_{2} & =H_{2}+\gamma H_{1} & \\
q_{3} & =H_{3}+(\beta / \alpha) H_{2} & \\
q_{4} & =H_{4}+\alpha H_{3}+\beta H_{2} & (n>4) \\
q_{n} & =H_{n}
\end{array}
$$

where $\alpha, \beta$ and $\gamma$ will be determined. Now it is not hard to see that for almost all $\alpha$ and $\beta$ there is a $\gamma$ that makes the above set of polynomials satisfy a 4 -term recursion. In fact if

$$
\begin{aligned}
\alpha & \neq 0 \\
6 \alpha^{2}-\beta(\beta+8) & \neq 0
\end{aligned}
$$

then

$$
\gamma=\frac{4 \alpha \beta}{6 \alpha^{2}-\beta(\beta+8)}
$$

works. It is also clear that

$$
\begin{aligned}
& \mathcal{L}\left[q_{0}\right]=1 \\
& \mathcal{L}\left[q_{n}\right]=0
\end{aligned} \quad(n>0) .
$$

That is $\mathcal{L}$ is the linear form for the 4 -term recursion. Finally, for almost any complex number $z_{0}$ we can find $\alpha$ and $\beta$ such that $q_{4}\left(z_{0}\right)=0$. It is possible that the $\alpha$ and $\beta$ found might not have an associated $\gamma$. However, if we then perturb $\alpha$ and $\beta$ then $q_{4}$ will have a zero near $z_{0}$. Thus we can guarantee a 4 -term recursion for which $q_{4}$ has complex roots.

However, to make things explicit, the following values work:

$$
\begin{aligned}
\alpha & =-4 / 3 \\
\beta & =28 / 3 \\
\gamma & =28 / 85 \\
q_{4}((1+i) / 2) & =0 .
\end{aligned}
$$

\section{Certain Macdonald-type Integrals}

We take the moments $\mu_{n}=\mu_{n}(C)$ with $C=3$ as defined in the form

$$
\mu_{n}=3^{n+4} \frac{\sqrt{3}}{2 \pi} \int_{0}^{1} f^{n}(v) g(v) d v
$$

where $f$ and $g$ are as given in (16), to obtain an expression for the determinants $\Delta_{n}=$ $\operatorname{det}\left[\mu_{i+j}\right]_{0 \leq i, j \leq n}$ as Macdonald-type integrals. Let

$$
I_{n}=\left(\frac{\sqrt{3}}{2 \pi}\right)^{n+1} \frac{3^{(n+1)(3 n+4)}}{(n+1) !} \int_{I^{(n+1)}} \prod_{0 \leq i<j \leq n}\left(v_{i}-v_{j}\right)^{2} \prod_{i=0}^{n} g\left(u_{i}\right) d u_{i}
$$


where

$$
\begin{aligned}
v_{i} & =u_{i}\left(1-u_{i}\right) \\
g\left(u_{i}\right) & =u_{i}^{\frac{1}{3}}\left(1-u_{i}\right)^{\frac{2}{3}}\left(1-2 u_{i}\right)
\end{aligned}
$$

We have

Theorem 3 If $\Delta_{n}=\operatorname{det}\left[\mu_{i+j}\right]_{0 \leq i, j \leq n}$, then $\Delta_{n}=I_{n}$.

Proof We put

$$
\begin{aligned}
B & =3^{4} \frac{\sqrt{3}}{2 \pi} \\
g(u) & =u^{\frac{1}{3}}(1-u)^{\frac{2}{3}}(1-2 u) \\
f(u) & =9 u(1-u)-1
\end{aligned}
$$

Then choosing $n+1$ variables $u_{0}, u_{1}, \ldots, u_{n}$, and labeling $f_{i}=f\left(u_{i}\right)$ and $g_{i}=g\left(u_{i}\right)(0 \leq i \leq n)$, we get

$$
\begin{aligned}
\Delta_{n} & =\operatorname{det}\left[\mu_{i+l}\right]_{0 \leq i, l \leq n} \\
& =B^{n+1} \int_{I^{(n+1)}} \prod_{i=0}^{n}\left(3 f_{i}\right)^{i} \operatorname{det}\left[\left(3 f_{k}\right)^{l}\right] \prod_{i=0}^{n} g_{i} d u_{i} \\
& =B^{n+1} 3^{n(n+1)} \int_{I^{(n+1)}} \prod_{i=0}^{n}\left(f_{i}\right)^{i} \operatorname{det}\left[f_{k}^{l}\right] \prod_{i=0}^{n} g_{i} d u_{i}
\end{aligned}
$$

where the integral is over the $(n+1)$-fold product of the unit interval. Now sum over all $(n+1)$ ! permutations of the indices to get

$$
\begin{aligned}
(n+1) ! \Delta_{n} & =B^{n+1} 3^{n(n+1)} \int_{I^{(n+1)}} \operatorname{det}\left[f_{k}^{l}\right]^{2} \prod_{i=0}^{n} g_{i} d u_{i} \\
& =B^{n+1} 3^{n(n+1)} \int_{I^{(n+1)}}\left[\prod_{0 \leq i<j \leq n}\left(f_{i}-f_{j}\right)\right]^{2} \prod_{i=0}^{n} g_{i} d u_{i} \\
& =B^{n+1} 3^{3 n(n+1)} \int_{I^{(n+1)}} \prod_{0 \leq i<j \leq n}\left(v_{i}-v_{j}\right)^{2} \prod_{i=0}^{n} g_{i} d u_{i}
\end{aligned}
$$

where

$$
v_{i}=u_{i}\left(1-u_{i}\right)
$$

The theorem follows immediately.

From what is said in the next several sections, it is reasonable to think that the $\Delta_{n}$ give the number of ASMs with vertical symmetry. In that case, these integrals should also count the ASMs with vertical symmetry. 
For example, expand

$$
\prod_{0 \leq i<j \leq n}\left(v_{i}-v_{j}\right)^{2}=\sum_{(i)} c_{i_{0}, i_{1}, \cdots, i_{n}} v_{0}^{i_{0}} v_{1}^{i_{1}} \cdots v_{n}^{i_{n}}
$$

where the coefficients are integers and the sum is over compositions $(i)=\left(i_{0}, i_{1}, \ldots, i_{n}\right)$ of $n(n+1)$ in which $0 \leq i_{k} \leq 2 n$ for every $k$. Substituting into the integral and noting that

$$
\int_{0}^{1} u^{\alpha-1}(1-u)^{\beta-1}(1-2 u) d u=\frac{(\beta-\alpha)}{\alpha} \cdot \frac{\Gamma(\alpha+1) \Gamma(\beta)}{\Gamma(\alpha+\beta+1)}
$$

and in the case $\alpha=i_{k}+\frac{4}{3}$, and $\beta=i_{k}+\frac{5}{3}$,

$$
\frac{(\beta-\alpha)}{\alpha} \cdot \frac{\Gamma(\alpha+1) \Gamma(\beta)}{\Gamma(\alpha+\beta+1)}=\frac{1}{3^{3 i_{k}+4}} \cdot \frac{2 \pi}{\sqrt{3}} \cdot \frac{\Gamma\left(3 i_{k}+4\right)}{\Gamma\left(2 i_{k}+4\right) \Gamma\left(i_{k}+2\right)}
$$

we arrive at

$$
(n+1) ! \Delta_{n}=\sum_{(i)} c_{i_{0}, i_{1}, \cdots, i_{n}} \prod_{k=0}^{n} M_{i_{k}}
$$

where

$$
M_{k}=\frac{1}{k+1}\left(\begin{array}{c}
3 k+3 \\
k
\end{array}\right)
$$

In order to evaluate the sum, we may assume that the indices form a partition of $n(n+1)$, in which case $0 \leq i_{0} \leq i_{1} \leq \cdots \leq i_{n}$ and the $i_{k}$ run over the range $k \leq i_{k} \leq n+k$. This change will only involve a new collection of integer coefficients for which we keep the same notation. Thus if $n=0$, there is one term with

$$
i_{0}=0 \quad c_{0}=1
$$

and

$$
\Delta_{0}=\left(\begin{array}{l}
3 \\
0
\end{array}\right)=1
$$

If $n=1$, the original sum over

$$
\begin{aligned}
\left(i_{0}, i_{1}\right) & =(0,2) \quad c_{0,2}=1 \\
& =(2,0) \quad c_{2,0}=1 \\
& =(1,1) \quad c_{1,1}=-2
\end{aligned}
$$

is replaced with a sum over

$$
\begin{aligned}
\left(i_{0}, i_{1}\right) & =(0,2) \quad c_{0,2}=2 \\
& =(1,1) \quad c_{1,1}=-2
\end{aligned}
$$

and dividing by 2 ! we get

$$
\begin{aligned}
\Delta_{1} & =\operatorname{det}\left[M_{i+j}\right]_{0 \leq i \leq j \leq 1} \\
& =M_{0} M_{2}-M_{1}^{2} \\
& =3
\end{aligned}
$$


In the case $n=2$, the sum is over

$$
\begin{aligned}
\left(i_{0}, i_{1}, i_{2}\right) & =(0,2,4) \quad c_{(i)}=6 \\
& =(0,3,3) \quad c_{(i)}=-6 \\
& =(1,1,4) \quad c_{(i)}=-6 \\
& =(1,2,3) \quad c_{(i)}=12 \\
& =(2,2,2) \quad c_{(i)}=-6
\end{aligned}
$$

so that after dividing by 3 !, we get

$$
\begin{aligned}
\Delta_{2} & =\operatorname{det}\left[M_{i+j}\right]_{0 \leq i \leq j \leq 2} \\
& =M_{0} M_{2} M_{4}-M_{0} M_{3}^{2}-M_{1}^{2} M_{4}+2 M_{1} M_{2} M_{3}-M_{2}^{3} \\
& =26 .
\end{aligned}
$$

\section{Equivalent forms for $\Delta_{n}$}

Recall that

$$
\Delta_{n}(C)=\operatorname{det}\left[\mu_{i+j}(C)\right]_{0 \leq i, j \leq n}
$$

where the moments $\mu_{k}$ are defined by (13) and characterized in a variety of forms by Theorem 2. The determinant $\Delta_{n}(3)$ itself can be expressed in a number of different forms as the following theorem shows.

Theorem $4 \Delta_{n}(3)=\operatorname{det}\left[a_{i+j}\right]_{0 \leq i, j \leq n}$, where $a_{k}$ has any of the forms

1. $a_{k}=\frac{1}{k+1}\left(\begin{array}{c}3 k+3 \\ k\end{array}\right)$,

2. $a_{k}=b_{k}(x)=\sum_{j=0}^{k} \frac{j+1}{k+1}\left(\begin{array}{c}3 k-j+1 \\ k-j\end{array}\right) x^{j}$ for any $x$. In particular $\operatorname{det}\left[b_{i+j}(x)\right]_{0 \leq i, j \leq n}$ evaluates to $\Delta_{n}(3)$ independently of $x$.

3. $a_{k}=\frac{1}{k+1}\left(\begin{array}{c}3 k+1 \\ k\end{array}\right)$,

4. $a_{k}=\left(\begin{array}{c}3 k+2 \\ k\end{array}\right)$.

Proof We will first deal with the case where

$$
a_{k}=\frac{1}{k+1}\left(\begin{array}{c}
3 k+3 \\
k
\end{array}\right) \text {. }
$$

We will use the following expression for $\mu_{k}$ from Theorem 2 ,

$$
\mu_{k}=\frac{\sqrt{3}}{2 \pi} 3^{k+4} \int_{0}^{1} f^{k}(u) g(u) d u
$$

where

$$
\begin{aligned}
& f(u)=9 u(1-u)-1 \\
& g(u)=u^{\frac{1}{3}}(1-u)^{\frac{2}{3}}(1-2 u)
\end{aligned}
$$


as in (16). This form for the $\mu_{k}$ allows us to make the following computation:

$$
\begin{aligned}
\sum_{i=0}^{k}\left(\begin{array}{c}
k \\
i
\end{array}\right) 3^{k-i} \mu_{i} & =\frac{\sqrt{3}}{2 \pi} 3^{k+4} \int_{0}^{1}(f(u)+1)^{k} g(u) d u \\
& =\frac{\sqrt{3}}{2 \pi} 3^{3 k+4} \int_{0}^{1}(1-2 u) u^{k+\frac{1}{3}}(1-u)^{k+\frac{2}{3}} g(u) d u \\
& =\frac{\sqrt{3}}{2 \pi} 3^{3 k+4}\left(\frac{\Gamma\left(k+\frac{4}{3}\right) \Gamma\left(k+\frac{5}{3}\right)}{\Gamma(2 k+3)}-2 \frac{\Gamma\left(k+\frac{7}{3}\right) \Gamma\left(k+\frac{4}{3}\right)}{\Gamma(2 k+4)}\right) \\
& =\frac{\sqrt{3}}{2 \pi} 3^{3 k+4}\left(2 k+3-2\left(k+\frac{4}{3}\right)\right) \frac{\Gamma\left(k+\frac{4}{3}\right) \Gamma\left(k+\frac{5}{3}\right)}{\Gamma(2 k+4)} \\
& =\frac{\sqrt{3}}{2 \pi} 3^{3 k+3} \frac{\Gamma(3 k+3) 2 \pi}{\Gamma(2 k+4) \Gamma(k+1) 3^{3 k+3-\frac{1}{2}}} \\
& =\frac{1}{k+1}\left(\begin{array}{c}
3 k+3 \\
k
\end{array}\right) \\
& =a_{k}
\end{aligned}
$$

Now row and column manipulations show that the determinant of

$$
a_{i+j}=\sum_{k=0}^{i+j}\left(\begin{array}{c}
i+j \\
k
\end{array}\right) 3^{i+j-k} \mu_{k}
$$

is equal to the determinant of $\mu_{i+j}$. This proves part 1 .

We now prove part 2 . The first 5 polynomials $b_{k}(x)$ are

$$
\begin{aligned}
& b_{0}(x)=1 \\
& b_{1}(x)=x+2 \\
& b_{2}(x)=x^{2}+4 x+7 \\
& b_{3}(x)=x^{3}+6 x^{2}+18 x+30 \\
& b_{4}(x)=x^{4}+8 x^{3}+33 x^{2}+88 x+143
\end{aligned}
$$

The proof of 2 is related to the enumeration of 2-line arrays of positive integers

$$
\begin{array}{cccc}
e_{1} & e_{2} & \cdots & e_{k} \\
f_{1} & f_{2} & \cdots & f_{k}
\end{array}
$$

such that $1=f_{1}=e_{1} \leq f_{2} \leq e_{2} \leq \cdots \leq f_{k} \leq e_{k} \leq j$ and $e_{i} \leq i,(1 \leq i \leq k)$. Let $h_{k, j}$ be the number of such arrays. Carlitz proved [2] that

$$
h_{k, k-j+1}=\frac{j}{k}\left(\begin{array}{c}
3 k-j-1 \\
k-j
\end{array}\right), \quad(1 \leq j \leq k),
$$

and

$$
\frac{1}{k}\left(\begin{array}{c}
3 k \\
k-1
\end{array}\right)=\sum_{j=1}^{k} h_{k, k-j+1}
$$


We define the polynomials $b_{k}(x)$ in terms of the numbers $h_{k, j}$ of Carlitz as

$$
b_{k}(x)=x^{k+1} \sum_{j=1}^{k+1} h_{k+1, j} x^{-j} .
$$

It can be proved that the $b_{k}(x)$ satisfy the recursion

$$
(1-x)^{2} b_{k}(x)-x^{3} b_{k-1}(x)=\frac{1}{k+1}\left(\begin{array}{c}
3 k+1 \\
k
\end{array}\right)-\frac{2}{k+1}\left(\begin{array}{c}
3 k \\
k
\end{array}\right) x
$$

for $k \geq 1$ with $b_{0}(x)=1$ by comparing coefficients and verifying the resulting binomial identity. In particular there is a representation of the form

$$
(1-x)^{2} b_{k}(x)-x^{3} b_{k-1}(x)=\alpha_{k}-\beta_{k} x
$$

for two numerical sequences $\left\{\alpha_{k}\right\}$ and $\left\{\beta_{k}\right\}$ defined in (29). Now perform elementary row operations on the matrix $B_{n}=\left[b_{i+j}(x)\right]$ as follows: multiply the last row by $(1-x)^{2}$, and subtract from it $x^{3}$ times the $(n-1)$-st row. Then multiply the $(n-1)$-st row by $(1-x)^{2}$, and subtract from it $x^{3}$ times the $(n-2)$-nd row, and so on, continuing down to $n=1$. Only the first row stays $b_{0}(x), b_{1}(x), \ldots, b_{n-1}(x)$. All other entries of the transformed matrix are now of the form $\alpha_{r}-\beta_{r} x$, i.e. linear in $x$. Call this new matrix $C_{n}$. The operations on $B_{n}$ multiply the determinant by $(1-x)^{2 n}$ and so

$$
(1-x)^{2 n} \operatorname{det}\left(B_{n}\right)=\operatorname{det}\left(C_{n}\right)
$$

But $\operatorname{det}\left(C_{n}\right)$ is a polynomial of degree $n+n=2 n$ from first principles. Since $\operatorname{det}\left(B_{n}\right)$ is a polynomial in $x$, the left hand side of (30) is a polynomial of degree at least $2 n$, and therefore exactly $2 n$. This forces $\operatorname{det}\left(B_{n}\right)$ to be a constant independent of $x$. By Carlitz's summation in $(28)$,

$$
b_{k}(1)=\frac{1}{k+1}\left(\begin{array}{c}
3 k+3 \\
k
\end{array}\right)
$$

and therefore by part 1 of the Theorem, $\Delta_{n}=\operatorname{det}\left(B_{n}\right)$. Specializing $b_{k}(x)$ at $x=0$ gives

$$
b_{k}(0)=\frac{1}{k+1}\left(\begin{array}{c}
3 k+1 \\
k
\end{array}\right)
$$

which proves part 3, while part 4 is a consequence of the binomial identity

$$
b_{k}(3)=\left(\begin{array}{c}
3 k+2 \\
k
\end{array}\right)
$$

\section{Remark}

As we have seen in (25) and (26) of Section 5 for the first few values of $n$, the polynomials $\Delta_{n}(t+3)$ appear to have non-negative coefficients, the constant term agreeing with the number of ASMs 
with vertical symmetry. Consider the matrix entries in the equivalent forms for $\Delta_{n}$ given in Theorem 4. These can be interpreted as corresponding to the $t=0$ case of the determinant for any of the alternate formulations for $a_{k}$ in Theorem 4. For example $\Delta_{n}=\operatorname{det}\left[a_{i+j}\right]$ with $a_{k}=\frac{1}{3 k+1}\left(\begin{array}{c}3 k+1 \\ k\end{array}\right)$, and it is reasonable to assume that $a_{k}$ is the constant term in some polynomial $a_{k}(t)$, for which $\operatorname{det}\left[a_{i+j}(t)\right]$ is identical to the polynomial $\Delta_{n}(3+t)$ obtained through the $\mu_{k}(3+t)$ 's.

Theorem $5 \quad \Delta_{n}(3)=R R(n)$.

Proof For this proof we will use the representation of $\Delta_{n}(3)$ as the determinant of the matrix

$$
A_{n}=\left[\left(\begin{array}{c}
3(i+j)+2 \\
i+j
\end{array}\right)\right]_{0 \leq i, j \leq n}
$$

which is the interpretation of $\Delta_{n}(3)$ given in part 4 of Theorem 4 . In a private correspondence, we have learned that Ira Gessel and Guoce Xin have independently discovered a different approach to calculating this determinant. The approach we take is a variation of finding the LDU decomposition of the matrix $A_{n}$. More specifically we find a lower triangular matrix,

$$
\left[\begin{array}{llll}
w_{0,0} & & \\
w_{1,0} & w_{1,1} & & \\
& \ldots & & \\
w_{n, 0} & w_{n, 1} & \ldots & w_{n, n}
\end{array}\right]
$$

so that

$$
\left[\begin{array}{llll}
w_{0,0} & & & \\
w_{1,0} & w_{1,1} & & \\
& \ldots & & \\
w_{n, 0} & w_{n, 1} & \ldots & w_{n, n}
\end{array}\right]\left[\begin{array}{lll}
a_{0,0} & \ldots & a_{0, n} \\
a_{1,0} & \ldots & a_{1, n} \\
& \ldots & \\
a_{n, 0} & \ldots & a_{n, n}
\end{array}\right]
$$

is an upper triangular matrix where

$$
a_{i, j}=\left(\begin{array}{c}
3(i+j)+2 \\
i+j
\end{array}\right)
$$

We normalize the $w$-matrix so that $w_{i, i}=1$ for all $i \geq 0$. We then proceed to guess the formula for the $w$-matrix. This is given below:

$$
w_{i, j}=\sum_{k}\left[\frac{(-1)^{i+j+k}(3 k) !}{(2 k+1) ! k !}\left(\begin{array}{c}
2 i \\
i+j+k
\end{array}\right) \frac{(3 i+2) !(j+k) !(2 i+2 j+2 k+1) !}{(3 j+3 k+2) ! i !(4 i+1) !}\right] .
$$

We plan on providing more details on how this guess was obtained elsewhere. It can easily be checked to be accurate with matrices with sizes up to $30 \times 30$.

We then proceeded to use automated tools to validate this guess. For notational convenience we rename the running indices by $i$ and $j$, and denote the row and the column indices of the matrix by $n$ and $m$ respectively. Then we need to demonstrate that the following double sum 


$$
\begin{aligned}
\sum_{i=0}^{n} w_{m, i} a_{i, n} & = \\
& \sum_{i=0}^{n} \sum_{j=0}^{n-i}\left[\frac{(-1)^{m+i+j}(3 j) !}{(2 j+1) ! j !}\left(\begin{array}{c}
2 m \\
m+i+j
\end{array}\right) \frac{(3 m+2) !(i+j) !(2 m+2 i+2 j+1) !}{(3 i+3 j+2) ! m !(4 m+1) !}\left(\begin{array}{c}
3(i+n)+2 \\
i+n
\end{array}\right)\right]
\end{aligned}
$$

is zero if $m<n$ and is

$$
\frac{\left(\begin{array}{c}
6 n+4 \\
2 n+2
\end{array}\right)}{2\left(\begin{array}{c}
4 n+3 \\
2 n+2
\end{array}\right)}
$$

if $m=n$. These types of identities can be proved automatically using a tool due to Wilf and Zeilberger: see for example, Kurt Wegschaider's thesis [12] for a comprehensive treatment.

We rewrite the double sum, and equivalently show that for $m<n$ (below the diagonal)

$$
\sum_{i=0}^{n} \sum_{j=0}^{n-i} \frac{(-1)^{i+j}(3 j) !(i+j) !(2+3 i+3 m) !(1+2 i+2 j+2 n) !}{j !(1+2 j) !(2+3 i+3 j) !(i+m) !(2+2 i+2 m) !(-i-j+n) !(i+j+n) !}=0
$$

and for $n=m$ (on the diagonal)

$$
\sum_{i=0}^{n} \sum_{j=0}^{n-i} \frac{(-1)^{n+i+j}(3 j) !(3 n+3 i+2) !(i+j) !(2 n+2 i+2 j+1) !(3 n-1) !(4 n+3) !}{j !(2 j+1) !(n+i+j) !(n-i-j) !(n+i) !(2 n+2 i+2) !(3 j+3 i+2) !(n-1) !(2 n+1)(6 n+1) !}=1 .
$$

To incorporate the condition $m<n$, we multiply the summand in (31) by $\left(\begin{array}{c}n-1 \\ m\end{array}\right)$ and set

$$
F[m, n, i, j]=\frac{(-1)^{i+j}(3 j) !(i+j) !(2+3 i+3 m) !(1+2 i+2 j+2 n) !\left(\begin{array}{c}
n-1 \\
m
\end{array}\right)}{j !(1+2 j) !(2+3 i+3 j) !(i+m) !(2+2 i+2 m) !(-i-j+n) !(i+j+n) !}
$$

The following certificate for $F$ proving (31) was computed by Akalu Tefera:

$$
\begin{aligned}
& 12(-2+n)(-1+n)(1+4 n)(-7+6 n)(-5+6 n) F[-2+m,-2+n,-1+i, j]+ \\
& 12(m-n)(-1+n)(-1+4 n)\left(-4-9 n+18 n^{2}\right) F[-2+m,-1+n,-1+i, j]+ \\
& 3(-1+m-n)(m-n)(1+3 n)(2+3 n)(-3+4 n) F[-2+m, n,-1+i, j]+ \\
& 4(-1+m)(-1+n)(-3+4 n)(-1+4 n)(1+4 n) F[-1+m,-1+n,-1+i, j]= \\
& \Delta[i,-12(-2+n)(-1+n)(1+4 n)(-7+6 n)(-5+6 n) F[-2+m,-2+n,-1+i, j]- \\
& 12(m-n)(-1+n)(-1+4 n)\left(-4-9 n+18 n^{2}\right) F[-2+m,-1+n,-1+i, j]- \\
& 3(-1+m-n)(m-n)(1+3 n)(2+3 n)(-3+4 n) F[-2+m, n,-1+i, j]]+\Delta[j, 0]
\end{aligned}
$$

The certificate for the sum (32) with summand $F[n, i, j]$ given by

$$
\frac{(-1)^{n+i+j}(3 j) !(3 n+3 i+2) !(i+j) !(2 n+2 i+2 j+1) !(3 n-1) !(4 n+3) !}{j !(2 j+1) !(n+i+j) !(n-i-j) !(n+i) !(2 n+2 i+2) !(3 j+3 i+2) !(n-1) !(2 n+1)(6 n+1) !}
$$


turned out to be much more complicated. We ran Kurt Wegschaider's Mathematica program MultiSum with the command FindRecurrence [summand, $n, i, j, 1]$. The resulting certificate proving (32) can be accessed online ${ }^{1}$. It is safe to bet that this "one-line proof" of (32) is a record-setter as far as long certificates go, as the certificate file is over $1.2 \mathrm{MB}$, and contains about 20,000 lines.

\section{ASM, vertical symmetry, lattice path models}

\section{Alternating Sign Matrices}

An $n \times n$ matrix with entries from $\{-1,0,1\}$ is an Alternating Sign Matrix (ASM) if

1. every row and column has sum 1 ,

2. in every row and column, the non-zero entries start with 1 and alternate in sign.

Because of the second condition, the partial sums of elements of every row and column of an ASM must be 1 or 0 . Every permutation matrix is an ASM, and for $n=1,2$ these are the only ASMs. For $n=3$, there are 7 ASMs, the six $3 \times 3$ permutation matrices and the matrix

$$
\left[\begin{array}{rrr}
0 & 1 & 0 \\
1 & -1 & 1 \\
0 & 1 & 0
\end{array}\right]
$$

The first few values of the number of ASMs is

$$
1,2,7,42,429,7436, \ldots
$$

Numerous conjectures concerning ASMs were put forward by Mills, Robbins, and Rumsey in [7]. These are further described in [11]. A general formula for the number of $n \times n$ ASMs was conjectured by Mills-Robbins-Rumsey to be

$$
\prod_{k=0}^{n-1} \frac{(3 k+1) !}{(n+k) !}
$$

and proved by Zeilberger in 1996 [13]. Shorter proofs were subsequently given by Kuperberg [5] and a refinement by Zeilberger [14]. There is a substantial amount of combinatorics concerning ASMs that is still not fully understood. Proofs for the number of ASMs having symmetries (e.g. invariant under reflection about a vertical axis, invariant under a 90 degree rotation, etc.) for which there are conjectured formulas have only recently been announced by Greg Kuperberg [6]. For example, the number of $(2 n+3) \times(2 n+3) \mathrm{ASMs}^{2}$ symmetric about a vertical axis is

$$
R R(n)=\prod_{k=0}^{n} \frac{\left(\begin{array}{c}
6 k+4 \\
2 k+2
\end{array}\right)}{2\left(\begin{array}{c}
4 k+3 \\
2 k+2
\end{array}\right)}
$$

which starts out as 1,3,26,646, 45885 for $n=0,1,2,3,4$. A full account of the history of ASMs can be found in [1].

\footnotetext{
${ }^{1}$ http://www.cs.ucsb.edu/ omer/diagonal-certificate.txt

${ }^{2}$ For technical reasons we start with the $3 \times 3$ case in this paper.
} 


\section{Lattice Paths}

In this section we consider lattice paths in the plane with unit steps in the direction of the horizontal and vertical axes. The elementary steps are

$$
\begin{aligned}
& (a, b) \rightarrow(a+1, b) \quad \text { (horizontal step) } \\
& (a, b) \rightarrow(a, b+1) \quad \text { (vertical step) }
\end{aligned}
$$

Thus a $t$-step path $\pi$ is the union of the closed line segments determined by a sequence of distinct lattice points $\left(s_{0}, s_{1}, \ldots, s_{t}\right)$ such that if $s_{i}=\left(x_{i}, y_{i}\right)$, then $x_{i+1}-x_{i}$ and $y_{i+1}-y_{i}$ are in $\{0,1\}$. The path $\pi$ is said to be from $s_{0}$ to $s_{t}$, denoted by $\pi: s_{0} \rightarrow s_{t}$. Given lattice points $A=\left(a_{1}, a_{2}\right)$ and $B=\left(b_{1}, b_{2}\right)$, there is an encoding of a path $\pi: A \rightarrow B$ as a word over $\{1,2\}$ consisting of $b_{1}-a_{1}$ occurrences of 1 (horizontal steps) and $b_{2}-a_{2}$ occurrences of 2 (vertical steps). Thus the number of paths from $A$ to $B$ is the binomial coefficient

$$
\left(\begin{array}{c}
b_{1}-a_{1}+b_{2}-a_{2} \\
b_{1}-a_{1}
\end{array}\right)
$$

Note that this number is zero unless $B$ is weakly to the North-East of $A$. For sets of points $A_{i}=\left(a_{1}^{i}, a_{2}^{i}\right)$ and $B_{i}=\left(b_{1}^{i}, b_{2}^{i}\right),(1 \leq i \leq n)$, we single out two families of $n$-tuples of paths $\Pi=\left(\pi_{1}, \ldots, \pi_{n}\right)$ with $\pi_{i}: A_{i} \rightarrow B_{i}$. $\Pi$ is called

1. Non-intersecting if no two paths $\pi_{i}, \pi_{j}$ have a common point,

2. Osculating if the paths are allowed to meet at lattice points only, but without crossing.

Figure 7 shows a 4-tuple of non-intersecting paths, and the rightmost figure in Figure 5 shows a 5-tuple of osculating paths where the osculation points are indicated by circles. In all of these examples, the path from $A_{0}$ to $B_{0}$ is a degenerate path consisting of a single lattice point with no horizontal or vertical steps.

There is a standard tool for representing the number of non-intersecting families of paths as a determinant via involutions, assuming certain restrictions on the relative positions of the points $A_{i}$ and $B_{i}$. For a permutation $\sigma$ of the indices $i$, let $\Pi^{\sigma}$ denote $n$-tuples of paths $\Pi^{\sigma}=$ $\left(\pi_{1}^{\sigma}, \ldots, \pi_{n}^{\sigma}\right)$ with $\pi_{i}^{\sigma}: A_{i} \rightarrow B_{\sigma(i)}$ and set $\operatorname{sign}\left(\Pi^{\sigma}\right)=\operatorname{sign}(\sigma)$. Then

$$
\sum_{\sigma} \sum_{\Pi^{\sigma}} \operatorname{sign}\left(\Pi^{\sigma}\right)=\operatorname{det}\left[\left(\begin{array}{c}
a_{1}^{i}-b_{1}^{j}+a_{2}^{i}-b_{2}^{j} \\
a_{1}^{i}-b_{1}^{j}
\end{array}\right)\right]_{1 \leq i, j \leq n}
$$

For any involution on the unrestricted set of $n$-tuples of paths $\Pi^{\sigma}$ which is sign-reversing outside its fixed point set $\mathcal{F}$, the left-hand side of (36) can be written as

$$
\sum_{\Pi^{\sigma} \in \mathcal{F}} \operatorname{sign}\left(\Pi^{\sigma}\right)
$$

A sign-reversing involution can be defined by locating a canonical pair of intersecting paths (such as the smallest labeled intersection of the smallest pair in lexicographic order), and switching 
the portions of the paths after the intersection point. If the position of the points $A_{i}$ and $B_{i}$ guarantee that any $n$-tuple $\Pi^{\sigma}$ corresponding to a non-identity $\sigma$ is intersecting, such as when

$$
\begin{aligned}
a_{1}^{1} \leq a_{1}^{2} \leq \cdots \leq a_{1}^{n} \quad \text { and } \quad a_{2}^{1} \geq a_{2}^{2} \geq \cdots \geq a_{2}^{n}, \\
b_{1}^{1} \leq b_{1}^{2} \leq \cdots \leq b_{1}^{n} \quad \text { and } \quad b_{2}^{1} \geq b_{2}^{2} \geq \cdots \geq b_{2}^{n},
\end{aligned}
$$

then the determinant in (36) counts the number of $n$ tuples of non-intersecting paths $\Pi=$ $\left(\pi_{1}, \ldots, \pi_{n}\right)$ with $\pi_{i}: A_{i} \rightarrow B_{i}$.

\section{ASM and Osculating Paths}

The path interpretation of ASMs in terms of osculating paths is directly based on the corner sum matrix introduced by Robbins and Rumsey in [10]: Given an $n \times n$ matrix $A$, the corner sum matrix $\bar{A}$ of $A$ is defined by

$$
\bar{A}_{i, j}=\sum A_{k, l}
$$

where the sum is over all pairs of integers $(k, l)$ with $k \geq i$ and $l \geq j$ (the interpretation here differs from [10] only in the ordering of the row and column indices of $A$ ). $A_{k, l}$ is regarded as zero if $k$ or $l$ is out of the range $\{1,2, \ldots, n\}$. The differences $\bar{A}_{i, j}-\bar{A}_{i+1, j}$ and $\bar{A}_{i, j}-\bar{A}_{i, j+1}$ are the partial sums of the rows and columns of $A$. Using this observation the following characterization of ASMs in terms of corner sum matrices can be proved

Lemma 1 ([10], lemma 1) An $n \times n$ matrix $A$ is an ASM iff $\bar{A}$ satisfies

1. $\bar{A}_{1, i}=\bar{A}_{i, 1}=n+1-i$ for $i=1,2, \ldots, n$,

2. $\bar{A}_{i, j}-\bar{A}_{i, j+1}$ and $\bar{A}_{i, j}-\bar{A}_{i+1, j}$ are in $\{0,1\}$ for $1 \leq i, j \leq n$.

Therefore the corner-sum matrix $\bar{A}$ of an ASM $A$ has first row from right to left, and first column from bottom up the entries $1,2, \ldots, n$. The other entries $\bar{A}_{i, j}$ are from $\{0,1, \ldots, n\}$ so that the entry above and to the left is either equal to $\bar{A}_{i, j}$ or $1+\bar{A}_{i, j}$.

We think of $\bar{A}$ as consisting of $n^{2}$ cells in the $(n+1) \times(n+1)$ grid with label of the cell $(i, j)$ equal to the entry $\bar{A}_{i, j}$. Tracing the south and east boundaries of the cells in $\bar{A}$ corresponding to each fixed $i \in\{1,2, \ldots, n\}$ produces $n$ lattice paths Since the steps of the paths in the first row (a vertical step) and in the first column (a horizontal step) are predetermined by condition 1 of lemma 1 , these can be viewed as paths in the $n \times n$ grid. The rows start with the points $A_{0}, A_{1}, \ldots, A_{n-1}$ from top to bottom, and the columns with the points $B_{0}, B_{1}, \ldots, B_{n-1}$ Let $\Pi=\left(\pi_{0}, \pi_{1}, \ldots, \pi_{n-1}\right)$ where $\pi_{i}: A_{i} \rightarrow B_{i}$ is the lattice path obtained from the boundary of the entries $n-i$ in $\bar{A}$. Then the path from $A_{0}$ to $B_{0}$ is a single point, and the family $\Pi$ is osculating.

Next, we consider ASM with vertical symmetry. The path interpretation that accompanies Robbins and Rumsey's corner-sum matrix can again be interpreted as an osculating path model.

Definition $1 O_{n}$ denotes the number of $(n+1)$-tuples of osculating paths $\Pi=\left(\pi_{0}, \pi_{1}, \ldots, \pi_{n}\right)$ where

$$
A_{i}=(0,-2 i), \quad B_{i}=(i, 0),
$$

and $\pi_{i}: A_{i} \rightarrow B_{i}$ for $i=0,1, \ldots, n$. 
An example of such a 5 -tuple $\Pi$ family appears on the right hand side of Figure 5 for $n=4$.

Lemma $2 O_{n}$ is the number of $(2 n+3) \times(2 n+3)$ ASM with vertical symmetry.

Proof There is a simple one-to-one correspondence. Label the columns of $A$ as the $n+1$ left columns $L_{1}, \ldots, L_{n+1}$, the central column $c$ and the $n+1$ columns to the right of $c$ as $R_{1}, \ldots, R_{n+1}$. The proof of the lemma makes use of the corner-sum matrix interpretation for the general ASM, applied to the $(2 n+3) \times(n+2)$ matrix that consists of $c$ and $R_{1}, \ldots, R_{n+1}$. First of all note that the entries in $c$ must be $1,-1,1,-1, \ldots,-1,1$ by vertical symmetry. Furthermore the row sums in $R_{1}, \ldots, R_{n+1}$ must alternately be 0 or 1 from top to bottom. These conditions force that the entries in $c$ and $R_{1}, \ldots, R_{n+1}$ in $\bar{A}$ satisfy

1. In $c$, the entries of $\bar{A}$ from bottom up are $1,1,2,2, \ldots, n+1, n+1, n+2$.

2. In $R_{1}$, the entries of $\bar{A}$ from bottom up are $0,1,1,2,2, \ldots, n+1, n+1$.

3. In the first two rows in $R_{1}, R_{2}, \ldots, R_{n+1}$ the entries of $\bar{A}$ from right to left are $1,2,3, \ldots, n+$ 1.

4. In the last row in $R_{1}, R_{2}, \ldots, R_{n+1}$ the entries of $\bar{A}$ are 0 .

These properties imply that the paths that are obtained from the boundaries of the cells labeled $\{1,2, \ldots, n+1\}$ from $\bar{A}$ as in the case of the general ASM are now predetermined in the first two rows, the last row, and the columns $c$ and $R_{1}$. This leaves a $2 n \times n$ grid defining the points $A_{i}$ and $B_{i}$ as in Figure 5. The boundary of the cells labeled $n+1-i$ defines a path from $A_{i}$ to $B_{i}$ as given in (38). The path $\pi_{0}: A_{0} \rightarrow B_{0}$ is a degenerate path with one point, and the family $\Pi=\left(\pi_{0}, \pi_{1}, \ldots, \pi_{n}\right)$ is osculating. The reverse of this map is straightforward.

\begin{tabular}{r|rrrrr}
1 & 0 & 0 & 0 & 0 & 0 \\
-1 & 1 & 0 & 0 & 0 & 0 \\
1 & -1 & 0 & 1 & 0 & 0 \\
-1 & 1 & 0 & -1 & 0 & 1 \\
1 & -1 & 1 & 0 & 0 & 0 \\
-1 & 1 & -1 & 1 & 0 & 0 \\
1 & 0 & 0 & -1 & 1 & 0 \\
-1 & 0 & 0 & 1 & 0 & 0 \\
1 & -1 & 1 & 0 & 0 & 0 \\
-1 & 1 & 0 & 0 & 0 & 0 \\
1 & 0 & 0 & 0 & 0 & 0
\end{tabular}

\begin{tabular}{|l|llll|l|l|}
\hline 6 & 5 & 4 & 3 & 2 & 1 \\
5 & 5 & 4 & 3 & 2 & 1 \\
5 & 4 & 4 & 3 & 2 & 1 \\
4 & 4 & 3 & 2 & 2 & 1 \\
\hline 4 & 3 & 3 & 2 & 1 & 0 \\
3 & 3 & 2 & 1 & 1 & 0 \\
3 & 2 & 2 & 1 & 1 & 0 \\
2 & 2 & 2 & 1 & 0 & 0 \\
2 & 1 & 1 & 0 & 0 & 0 \\
1 & 1 & 0 & 0 & 0 & 0 \\
1 & 0 & 0 & 0 & 0 & 0 \\
\hline
\end{tabular}

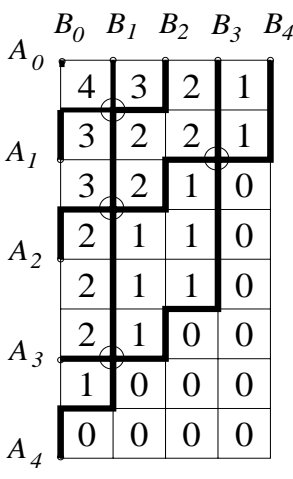

Figure 5: The columns $c, R_{1}, R_{2}, R_{3}, R_{4}, R_{5}$ of an $11 \times 11$ vertically symmetric ASM, the corner sum matrix, and the corresponding 5 -tuple of osculating paths. 
This construction is displayed in Figure 5 for the $11 \times 11$ vertically symmetric ASM on the left of the figure (the rightmost 6 columns are shown). The resulting 5 -tuple of osculating paths are as given on the right hand side.

\section{Path Interpretations \& Hankel Determinants}

Our path model for Hankel determinants related to ASM with vertical symmetry is as follows. Consider the set of points $A_{0}, A_{1}, \ldots, A_{n}$ and $B_{0}, B_{1}, \ldots, B_{n}$ where

$$
A_{i}=(-i,-2 i), \text { and } \quad B_{i}=(i, 2 i+2), \quad(0 \leq i \leq n)
$$

as displayed in Figure 6 for $n=3$. The points $A_{i}$ are on the line $y=2 x$ in the third quadrant,

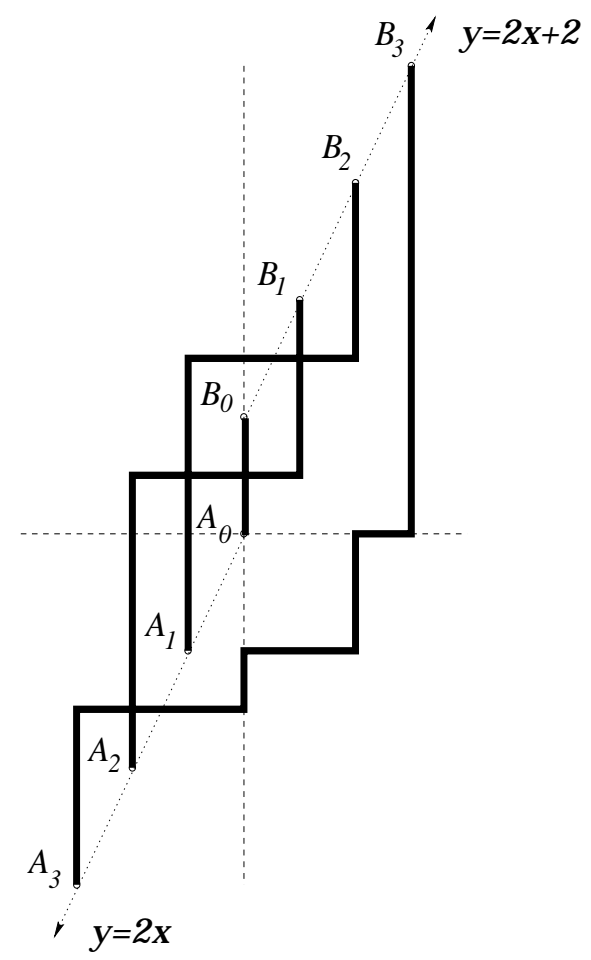

Figure 6: Path model for the $(n+1) \times(n+1)$ Hankel matrix (39) for $r=2$ and $n=3$. and the points $B_{i}$ are on the line $y=2 x+2$ in the first quadrant. The number of paths $\pi: A_{i} \rightarrow B_{j}$ is given by

$$
\left(\begin{array}{c}
3(i+j)+2 \\
i+j
\end{array}\right)
$$

which is the $(i, j)$-th entry of the Hankel matrix $\left[a_{i+j}\right]_{0 \leq i, j \leq n}$ with determinant $\Delta_{n}(3)$ we considered before with $a_{k}=\left(\begin{array}{c}3 k+2 \\ k\end{array}\right)$ (this is the characterization in part 4 of Theorem 4 ). In the general 
case, instead of the lines $y=2 x$ and $y=2 x+2$, we consider the lines $y=r x$ and $y=r x+r$ for $r \geq 1$ with

$$
A_{i}=(-i,-r i), \text { and } \quad B_{i}=(i, r i+r), \quad(0 \leq i \leq n) .
$$

In this way we obtain a path model for the $(n+1) \times(n+1)$ Hankel matrix

$$
\left[\left(\begin{array}{c}
(r+1)(i+j)+r \\
i+j
\end{array}\right)\right] .
$$

Our results hold for general $r$ in this setup, where the ASM with vertical symmetry corresponds to the case $r=2$.

Theorem 6 For any integer $r \geq 1$, det $\left[\left(\begin{array}{c}(r+1)(i+j)+r \\ i+j\end{array}\right)\right]_{0 \leq i, j \leq n}$ is the number of non-intersecting tuples of paths $\Pi=\left(\pi_{0}, \pi_{1}, \ldots, \pi_{n}\right)$ with $\pi_{i}: A_{i} \rightarrow C_{i}$ where

$$
A_{i}=(-i,-r i), \text { and } \quad C_{i}=(i,-i), \quad(0 \leq i \leq n)
$$

as shown in Figure 7 for $r=2$ and $n=3$.

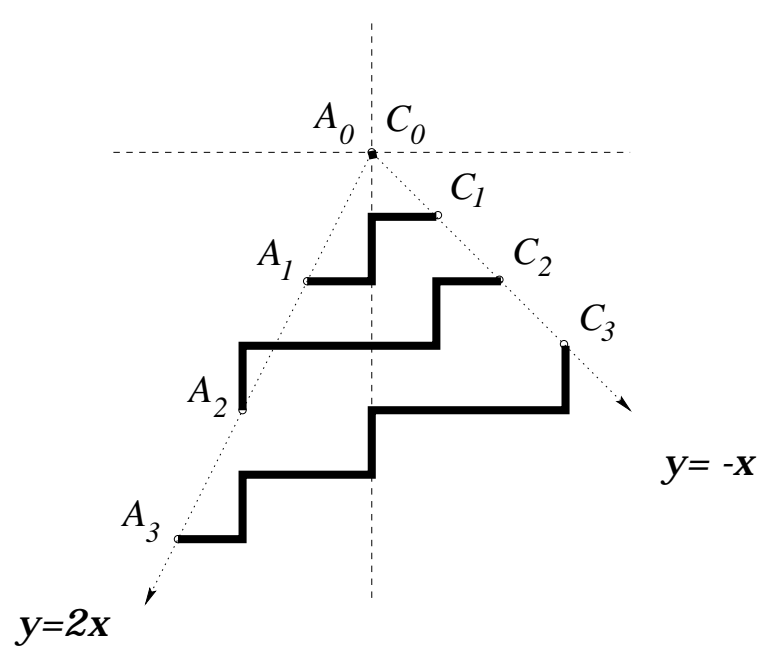

Figure 7: Tuples of non-intersecting paths (Hankel paths) counted by the Hankel determinant $\operatorname{det}\left[\left(\begin{array}{c}(r+1)(i+j)+r \\ i+j\end{array}\right)\right]$ for $r=2$ and $n=3$.

Proof First we work with tuples of paths from points $A_{i}$ to $B_{j}$ in Figure 6, and subsequently identify the tuples enumerated by the determinant as non-intersecting paths from points $A_{i}$ to $C_{i}$ in Figure 7. Note that the usual cancellation of intersecting $n$-tuples via sign reversing involutions fails for the placement of points Figure 6 , since it is possible to have non-identity permutations $\sigma$ for which $\Pi^{\sigma}$ is non-intersecting. However the interpretation (37) for the determinant still holds. We show that all tuples of paths can be paired up to cancel out except for those $\Pi=$ 
$\left(\pi_{0}, \pi_{1}, \ldots, \pi_{n}\right)$ corresponding to the identity permutation for which $\pi_{i}$ stays strictly to the North-West of $\pi_{j}$ whenever $i<j$. Assuming for a moment that we can do this, it is easy to see that we can identify such a family with paths from $A_{i}$ to $C_{i}$ instead of the $B_{i}$ as claimed in the Theorem, since the portion of $\pi_{i}$ from $C_{i}$ to $B_{i}$ must consist of vertical steps only for every $i$.

We proceed in two steps. First we pair up tuples $\Pi^{\sigma}$ in which some path $\pi_{i}: A_{i} \rightarrow B_{\sigma(i)}$ starts out by $r$ (or more) vertical steps (and therefore intersects the line $y=r x+r$ at $x=-i$ ). Consider any bijection $\alpha$ for the trivial binomial identity

$$
\left(\begin{array}{c}
(r+1) k \\
k
\end{array}\right)=(r+1)\left(\begin{array}{c}
(r+1) k-1 \\
k-1
\end{array}\right) .
$$

Locate the largest index $i$ in $\Pi^{\sigma}$ for which $\pi_{i}$ passes through $(-i,-r i+r)$, i.e. starts with $r$ vertical steps. Suppose $\sigma(i)=k$ and $\sigma(i-1)=l$ so that $\pi_{i}:(-i,-r i+r) \rightarrow B_{k}, \pi_{j}: A_{i-1} \rightarrow B_{l}$. Let $N=i+k$ and $M=i+l$. The number of such $\pi_{i}, \pi_{i-1}$ pairs is

$$
\left(\begin{array}{c}
(r+1) N \\
N
\end{array}\right)\left(\begin{array}{c}
(r+1) M-1 \\
M-1
\end{array}\right)
$$

Switching the endpoints, the number of pairs $\pi_{i}:(-i,-r i+r) \rightarrow B_{l}, \pi_{j}: A_{i-1} \rightarrow B_{k}$ is

$$
\left(\begin{array}{c}
(r+1) M \\
M
\end{array}\right)\left(\begin{array}{c}
(r+1) N-1 \\
N-1
\end{array}\right)
$$

and these latter pairs of paths have signs opposite to those of the former. Now $\alpha$ can be used to construct a bijection between these two sets of opposite signed pairs of paths by setting up a correspondence for

$$
\left(\begin{array}{c}
(r+1) N \\
N
\end{array}\right)\left(\begin{array}{c}
(r+1) M-1 \\
M-1
\end{array}\right)=(r+1)\left(\begin{array}{c}
(r+1) N-1 \\
N-1
\end{array}\right)\left(\begin{array}{c}
(r+1) M-1 \\
M-1
\end{array}\right)
$$

and

$$
\left(\begin{array}{c}
(r+1) M \\
M
\end{array}\right)\left(\begin{array}{c}
(r+1) N-1 \\
N-1
\end{array}\right)=(r+1)\left(\begin{array}{c}
(r+1) M-1 \\
M-1
\end{array}\right)\left(\begin{array}{c}
(r+1) N-1 \\
N-1
\end{array}\right)
$$

and composing the two bijections. Let $\mathcal{F}$ be the fixed point of this sign-reversing involution.

We can now consider only $\Pi^{\sigma} \in \mathcal{F}$. These are tuples $\Pi^{\sigma}$ in which no $\pi_{i}$ starts with $r$ vertical steps. Now by the pigeonhole principle, either $\pi_{i}$ stays North-West of $\pi_{j}$ in such a $\Pi^{\sigma}$ for all pairs $i<j$, or $\Pi^{\sigma}$ is an intersecting tuple of paths. Such intersecting ones in $\mathcal{F}$ can be canceled out by a standard sign-reversing involution, leaving the non-intersecting ones (such as the example in Figure 7) corresponding to the identity permutation as the tuples of paths enumerated by the determinant.

Definition $2 P_{n}$ denotes the number of (n+1)-tuples of non-intersecting paths $\Pi=\left(\pi_{0}, \pi_{1}, \ldots, \pi_{n}\right)$ with $\pi_{i}: A_{i} \rightarrow C_{i}$ where

$$
A_{i}=(-i,-2 i), \text { and } \quad C_{i}=(i,-i), \quad(0 \leq i \leq n),
$$


An example 4-tuple counted by $P_{n}$ is shown in Figure 7 for $r=2$ and $n=3$.

As a corollary of Theorem 6 with $r=2$, we have that

$$
P_{n}=\operatorname{det}\left[\left(\begin{array}{c}
3(i+j)+2 \\
i+j
\end{array}\right)\right]_{0 \leq i, j \leq n}
$$

and therefore the sequence $P_{n}$ starts out as $1,3,26,646,45885$ for $n=0,1,2,3,4$.

\section{Remark}

The values of the Hankel determinants in Theorem 6 for small values of $n$ and $r$ are given in Figure 8 . The second row is given by $R R(n)$ for $n=0,1,2,3,4$. For the first row, it is easy to

\begin{tabular}{|c||c|c|c|c|c|}
\hline$r \backslash n$ & 0 & 1 & 2 & 3 & 4 \\
\hline \hline 1 & 1 & 1 & 1 & 1 & 1 \\
\hline 2 & 1 & 3 & 26 & 646 & 45885 \\
\hline 3 & 1 & 6 & 206 & 40083 & 44042301 \\
\hline 4 & 1 & 10 & 950 & 848465 & 7096349476 \\
\hline 5 & 1 & 15 & 3200 & 9604260 & 403895099151 \\
\hline
\end{tabular}

Figure 8: Values of $\operatorname{det}\left[\left(\begin{array}{c}(r+1)(i+j)+r \\ i+j\end{array}\right)\right]_{0 \leq i, j \leq n}$ for small values of $r$ and $n$.

see from the path interpretation that

$$
\operatorname{det}\left[\left(\begin{array}{c}
2(i+j)+1 \\
i+j
\end{array}\right)\right]=1
$$

for every $n$ since there is only one tuple of paths $\Pi$ enumerated by the determinant, i.e. one in which each $\pi: A_{i} \rightarrow C_{i}$ consists of horizontal steps only. Since

$$
\operatorname{det}\left[\frac{1}{2(i+j)+1}\left(\begin{array}{c}
2(i+j)+1 \\
i+j
\end{array}\right)\right]=\operatorname{det}\left[\left(\begin{array}{c}
2(i+j)+1 \\
i+j
\end{array}\right)\right]
$$

we obtain a combinatorial proof of the well-known result that the Catalan-Hankel determinant on the right evaluates to unity [8].

\section{Remark}

Part of the bijection constructed for the proof of Theorem 6 was built upon $\alpha$ proving

$$
\left(\begin{array}{c}
c N \\
N
\end{array}\right)=c \cdot\left(\begin{array}{c}
c N-1 \\
N-1
\end{array}\right)
$$

Of course the equality of (40) and (41) follows from (42) trivially and does not require a bijection. In particular constructing a "nice" bijection $\alpha$ involves dividing combinatorially, which is usually problematic. This is best illustrated by the fact that the $q$-binomial identity

$$
\left(1+q+\cdots+q^{N-1}\right)\left[\begin{array}{c}
c N \\
N
\end{array}\right]=\left(1+q+\cdots+q^{N-1}\right)\left(1+q^{N}+\cdots+q^{(c-1) N}\right)\left[\begin{array}{c}
c N-1 \\
N-1
\end{array}\right]
$$


has a straightforward bijective proof, since both sides $q$-count the number of strings of type $1^{(c-1) N} 2^{N-1} 3^{1}$ by inversions, whereas

$$
\left[\begin{array}{c}
c N \\
N
\end{array}\right]=\left(1+q^{N}+\cdots+q^{(c-1) N}\right)\left[\begin{array}{c}
c N-1 \\
N-1
\end{array}\right]
$$

does not.

\section{Remark}

A related Hankel determinant which can easily be evaluated via a path model is $\operatorname{det}\left[a_{i+j}\right]_{0 \leq i, j \leq n}$ where $a_{k}=\left(\begin{array}{c}2 k \\ k\end{array}\right)$.

\section{Theorem 7}

$$
\operatorname{det}\left[\left(\begin{array}{c}
2(i+j) \\
i+j
\end{array}\right)\right]_{0 \leq i, j \leq n}=2^{n}
$$

Proof Consider the set of points $A_{0}, A_{1}, \ldots, A_{n}$ and $B_{0}, B_{1}, \ldots, B_{n}$ where

$$
A_{i}=(-i,-i), \text { and } \quad B_{i}=(i, i), \quad(0 \leq i \leq n)
$$

so that $A_{0}=B_{0}=(0,0)$. Then $a_{i+j}$ is the number of paths from $A_{i}$ to $B_{j}$, and the determinant can be interpreted as in (36). We show that $\operatorname{det}\left[a_{i+j}\right]_{0 \leq i, j \leq n}$ enumerates all $(n+1)$-tuples of paths $\Pi=\left(\pi_{0}, \pi_{1}, \ldots, \pi_{n}\right)$ corresponding to the identity permutation in which each $p_{i}$ is a rightangle between $A_{i}$ and $B_{i}$, i.e. each $\pi_{i}$ consists of horizontal steps followed by vertical steps, or vertical steps followed by horizontal steps as shown in Figure 9 for $n=4$. Since there are $2^{n}$

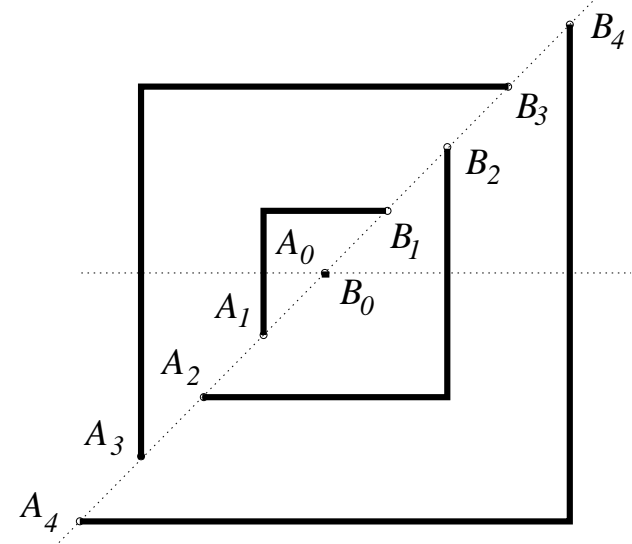

Figure 9: Path model for the Hankel determinant $\operatorname{det}\left[\left(\begin{array}{c}2(i+j) \\ i+j\end{array}\right)\right]_{0 \leq i, j \leq 4}$.

such $(n+1)$-tuples of paths, the Theorem will follow. The key observation is that for any two distinct points $B_{k}$ and $B_{l}$, the number of pairs of paths, one from $(-i,-j)$ to $B_{k}$ and one from $(-j,-i)$ to $B_{l}$ is

$$
\left(\begin{array}{c}
i+j+2 k \\
i+k
\end{array}\right)\left(\begin{array}{c}
i+j+2 l \\
j+l
\end{array}\right)
$$


and this can be written as

$$
\left(\begin{array}{c}
i+j+2 k \\
j+k
\end{array}\right)\left(\begin{array}{c}
i+j+2 l \\
i+l
\end{array}\right),
$$

which is the number of pairs of paths, one from $(-i,-j)$ to $B_{l}$ and one from $(-j,-i)$ to $B_{k}$. Note that in this case there is a trivial bijection between these two sets of pairs of paths that we can use as the part of an involution. We again proceed in two steps. First we pair up tuples $\Pi^{\sigma}$ in which some pair of paths $\pi_{r}: A_{r} \rightarrow B_{k}$ and $\pi_{s}: A_{s} \rightarrow B_{l}$ pass through symmetrically placed points $(-i,-j)$ and $(-j,-i)$, respectively. By the above observation, this pairing is sign reversing. Let $\mathcal{F}$ be the fixed point of this involution. On $\mathcal{F}$, we pair up intersecting pairs of paths in the usual way. The final fixed point set enumerated by the determinant consist of all $(n+1)$-tuples of paths $\Pi=\left(\pi_{0}, \pi_{1}, \ldots, \pi_{n}\right)$ such that

1. $\Pi$ is non-intersecting,

2. There are no two paths in $\Pi$ such that one passes through $(-i,-j)$, and the other through $(-j,-i)$ for any $i, j(-n \leq i, j \leq n)$.

Now notice that in any such tuple $\Pi$, we must have $\pi_{1}: A_{1} \rightarrow B_{1}$. Otherwise $\pi_{1}$ has to go through either $(2,-1)$, or $(-1,2)$, and in either case it is then impossible for any other path to reach $B_{1}$. Now $\pi_{2}: A_{2} \rightarrow B_{2}$, for otherwise $\pi_{2}$ has to pass through $(3,-2)$, or $(-2,3)$, making it impossible for any other path to reach $B_{2}$. It follows that $\pi_{i}: A_{i} \rightarrow B_{i}$ and each $\pi_{i}$ other than $\pi_{0}$ can be either type of a right-angle. This proves Theorem 7 .

\section{Remark}

There is a straightforward one-to-one correspondence between the Hankel paths counted by $P_{n}$ (as defined in Definition 2) and column-strict tableaux of staircase shape $(n+1, n, \ldots, 1)$ in which the entries in row $i$ are bounded by $2(n+1)-i$. For example, the 4 -tuple of nonintersecting paths in Figure 7 corresponds to the column strict tableau in Figure 10 where the bounds for the row entries are 7,6,5,4 from top to bottom. On the other hand, there is a

\begin{tabular}{|l|l|l|l|}
\hline 1 & 1 & 4 & 6 \\
\cline { 1 - 2 } 2 & 3 & 6 & \multicolumn{1}{|c}{} \\
\cline { 1 - 2 } 3 & 4 & \multicolumn{2}{|}{} \\
\cline { 1 - 2 } 4 & \multicolumn{2}{|}{} \\
\cline { 1 - 1 } & &
\end{tabular}

Figure 10: Column-strict tableau corresponding to the 4-tuple of paths in Figure 7.

one-to -one correspondence between the osculating paths corresponding to ASM with vertical symmetry counted by $O_{n}$ (as defined in Definition 1) and strict Gelfand patterns [11] with first row $1,3, \ldots, 2 n-1$. For example, the 5 -tuple of osculating paths in Figure 5 corresponds to the strict Gelfand pattern in Figure 11 with first row 1,3, 5, 7,9. 


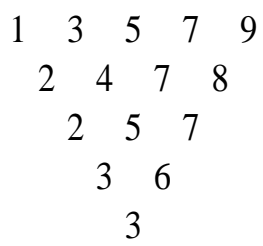

Figure 11: Strict Gelfand pattern corresponding to the 5-tuple of paths in Figure 5.

\section{Remark}

The two types of paths in question, the Hankel paths counted by $P_{n-1}$ (as defined in Definition 2 ) and the osculating paths corresponding to ASM with vertical symmetry counted by $O_{n-1}$ (as defined in Definition 1) can be shown to be in bijection with the families $\mathcal{F}_{1}(n)$ and $\mathcal{F}_{2}(n)$ of tableaux defined below. First, call a tableau (or a shifted tableau) with $n$ rows good if

1. the entries are bounded by $n$,

2. the first cell in row $i$ is labeled $i$,

3. entries are weakly-increasing down the anti-diagonals.

Let $\lambda=(2 n-1, \ldots, 3,1)$. Then $\mathcal{F}_{1}(n)$ is the family of column-strict good tableaux of shape $\lambda$, and $\mathcal{F}_{2}(n)$ is the family of shifted good tableaux of shape $\lambda$. Examples of these are given in Figures 12 and Figure 13 for $n=3$.

$\begin{array}{lllllllllllll}11111 & 11111 & 11111 & 11112 & 11112 & 11112 & 11113 & 11113 & 11113 & 11122 & 11122 & 11122 & 11123 \\ 222 & 223 & 233 & 222 & 223 & 233 & 222 & 223 & 233 & 222 & 223 & 233 & 222 \\ 3 & 3 & 3 & 3 & 3 & 3 & 3 & 3 & 3 & 3 & 3 & 3 & 3 \\ & & & & & & & & & & & & \\ 11123 & 11123 & 11133 & 11133 & 11222 & 11222 & 11223 & 11223 & 11233 & 11233 & 12222 & 12223 & 12233 \\ 223 & 233 & 223 & 233 & 223 & 233 & 223 & 233 & 223 & 233 & 233 & 233 & 233 \\ 3 & 3 & 3 & 3 & 3 & 3 & 3 & 3 & 3 & 3 & 3 & 3 & 3\end{array}$

Figure 12: Family $\mathcal{F}_{1}(3)$ of column-strict good tableaux of shape $(5,3,1)$ counted by $P_{2}$.

$\begin{array}{ccccccccccccc}11111 & 11111 & 11111 & 11112 & 11112 & 11112 & 11113 & 11113 & 11122 & 11122 & 11122 & 11123 & 11123 \\ 222 & 223 & 233 & 222 & 223 & 233 & 223 & 233 & 222 & 223 & 233 & 223 & 233 \\ 3 & 3 & 3 & 3 & 3 & 3 & 3 & 3 & 3 & 3 & 3 & 3 & 3 \\ & & & & & & & & & & & & \\ 11133 & 11222 & 11222 & 11222 & 11223 & 11223 & 11233 & 12222 & 12222 & 12222 & 12223 & 12223 & 12233 \\ 233 & 222 & 223 & 233 & 223 & 233 & 233 & 222 & 223 & 233 & 223 & 233 & 233 \\ 3 & 3 & 3 & 3 & 3 & 3 & 3 & 3 & 3 & 3 & 3 & 3 & 3\end{array}$

Figure 13: Family $\mathcal{F}_{2}(3)$ of shifted good tableaux of shape $(5,3,1)$ counted by $\mathrm{O}_{2}$. 


\section{Higher Order $\Delta_{n}$}

We note again that the Hankel determinants $\operatorname{det}\left[a_{i+j}\right]_{0 \leq i, j \leq n}$ with

$$
a_{k}=\left(\begin{array}{c}
2 k+1 \\
k
\end{array}\right) \text {, }
$$

all have the value 1 . It appears that the values $\operatorname{det}\left[a_{i+j}\right]_{0 \leq i, j \leq n}$ with

$$
a_{k}=\left(\begin{array}{c}
3 k+2 \\
k
\end{array}\right)
$$

are the same as the conjectured numbers for ASMs with vertical symmetry given by (34).

So in two cases, the values of an infinite class of determinants are given by smooth arithmetic sequences; i.e., the prime factors of the determinants come from ratios of products of binomial coefficients and are therefore small. This phenomenon seems to be quite extensive.

We now return to the critical value $C=6$ of section 3 , the value which specifies the polynomial sequence

$$
q_{n}=x q_{n-1}-6 q_{n-2}+4 q_{n-3}-q_{n-4}
$$

with initial polynomials $q_{-3}=q_{-2}=q_{-1}=0, q_{0}=1$ As in section 2 , we may define a linear functional $\mathcal{L}$ on the space of polynomials by

$$
\begin{aligned}
\mathcal{L}[1] & =1 \\
\mathcal{L}\left[q_{n}\right] & =0 \quad(n \geq 1) .
\end{aligned}
$$

This functional has moments $\mu_{n}=\mathcal{L}\left[x^{n}\right]$ the first seven values of which are

$$
\begin{aligned}
& \mu_{0}=1 \\
& \mu_{1}=0 \\
& \mu_{2}=6 \\
& \mu_{3}=-4 \\
& \mu_{4}=73 \\
& \mu_{5}=-120 \\
& \mu_{6}=1164 .
\end{aligned}
$$

The associated Hankel determinants $\Delta_{n}=\operatorname{det}\left[\mu_{i+j}\right]_{0 \leq i, j \leq n}$ begin

$$
\begin{aligned}
& \Delta_{0}=1 \\
& \Delta_{1}=6 \\
& \Delta_{2}=206 \\
& \Delta_{3}=40083 .
\end{aligned}
$$


It can readily be shown that these particular $\Delta_{n}$ can also be realized in other forms, and we mention $\Delta_{n}=\operatorname{det}\left[b_{i+j}\right]_{0 \leq i, j \leq n}$, where

$$
b_{k}=\left(\begin{array}{c}
4 k+3 \\
k
\end{array}\right) .
$$

In the tables, the largest prime factor of these determinants is generally quite large, and some further numerical data suggests that the Hankel determinants $\operatorname{det}\left[h_{i+j}\right]_{0 \leq i, j \leq n}$ with

$$
h_{k}=\left(\begin{array}{c}
a k+b \\
k
\end{array}\right) \quad(a \geq 4)
$$

are never smooth.

\section{Epilogue}

This paper began with the consideration of a certain transform of polynomials, which we have called the $T$-transform. From this transform we began our study of families of polynomials satisfying a Riemann hypothesis. These families occur as the terms generated by recursions, and except in the few cases where the recursions are 3-term, the empirical evidence of the existence of a Riemann hypothesis and of a familiar interlacing phenomenon cannot be studied within the framework of an existing theory. This led us to the consideration of renormalized recursions, out of which emerged several new problems, as well as a totally unexpected connection to various combinatorial objects, which include Alternating Sign Matrices with vertical symmetry. As to the new problems, we mention again the 3 -conjecture and the 6 -conjecture, which are merely two examples of infinitely many questions related to renormalized recursions and questions about the nature of interlacing phenomenon, as well as questions about the $t$-analogues generated by various Hankel determinant models presented.

We have indicated that to each renormalized recursion, there is a critical value, and that this critical value may be attached to rather interesting combinatorial questions. These combinatorial questions in turn may be approached from various different directions, which seem to unite problems in diverse areas. For example, it is unexpected that certain values of Macdonaldlike integrals we consider are associated to the number of ASMs with vertical symmetry. This integral formulation in turn, is a direct consequence of our interpretation of the values of certain Hankel determinants combinatorially by lattice path models in which the standard sign-reversing involutions do not apply.

Recently we have discovered a vast generalization of the $T$-transform, with the attendant Riemann hypothesis and interlacing phenomenon. This new twisted $T$-transform is a multidimensional convolution defined as follows. Given $\frac{n(n+1)}{2}$ positive integers

$$
\gamma=\left[\gamma_{i j}: 0 \leq i<j \leq n\right]
$$

we define a kernel via

$$
W[u ; \gamma, n]=\prod_{0 \leq i<j \leq n}\left[1-u_{i}-u_{j}\right]^{\gamma_{i j}}
$$

THE ELECTRONIC JOURNAL OF COMBINATORICS 8 (2001), \#R36 
Given $n+1$ polynomials

$$
P=\left[p_{k}: 0 \leq k \leq n\right],
$$

each having all their zeros on the line $\operatorname{Re}(s)=\frac{1}{2}$, their twisted transform is defined as

$$
T[P, \gamma](s)=\left(\frac{\sin \pi s}{\pi}\right)^{n+1} \int_{I^{(n+1)}} W[u ; \gamma, n] \prod_{k=0}^{n} p_{k}\left(u_{k}\right) u_{k}^{s}\left(1-u_{k}\right)^{1-s} \frac{d u_{k}}{u_{k}\left(1-u_{k}\right)} .
$$

The $T$-transform which led to the considerations in the present paper is the case $n=0$ of this with the empty product $W[u ; \gamma, n]=1$. For $T[P, \gamma]$, Redmond [9] was able to prove that

$$
p_{k} \in R h \text { for each } p_{k} \in P \Rightarrow T[P, \gamma](s) \in R h \text {. }
$$

However, the apparent interlacing phenomenon conjecture is still unproved. In a future paper we plan to address this question. Undoubtedly the general case will also raise a number of combinatorial questions and relations of the nature considered in the present paper. 


\section{References}

[1] D. Bressoud. Proofs and Confirmations: The Story of the Alternating Sign Matrix Conjecture. Cambridge University Press, Cambridge, England, 1999.

[2] L. Carlitz. "Enumeration of Two-line Arrays." Fibonacci Quarterly, 11 (1973), pp. 113-130.

[3] T. S. Chihara. Introduction to Orthogonal Polynomials. Gordon \& Breach Science Publishers, New York, 1978.

[4] Ö. Eğecioğlu and C. Ryavec. "Polynomial Families Satisfying a Riemann Hypothesis." Congressus Numerantium, 2002 (to appear).

[5] G. Kuperberg. "Another Proof of the Alternating-Sign Matrix Conjecture." Internat. Math. Res. Notes, No. 3 (1996), pp. 139-150.

[6] G. Kuperberg. "Symmetry classes of alternating-sign matrices under one roof." preprint, arXiv:math.Co/0008184, 24 August, 2000.

[7] W. H. Mills, D. P. Robbins, and H. Rumsey, Jr., "Alternating sign matrices and descending plane partitions." J. Combin. Theory Ser. A, 34 (1983), pp. 340-359.

[8] T. Muir. A Treatise on the Theory of Determinants. (revised and enlarged by W. H. Metzler), Dover Publications, New York, 1933.

[9] T. Redmond, "A Class of Polynomial Riemann Hypotheses," preprint.

[10] D. P. Robbins and H. Rumsey, Jr., "Determinants and Alternating Sign Matrices." Advances in Math., 62 (1986), pp. 169-184.

[11] R. P. Stanley. "A baker's dozen of conjectures concerning plane partitions," in Combinatoire Enumerative, Lecture Notes in Mathematics, Vol. 1234, New York: Springer-Verlag (1984), pp. 285-293.

[12] K. Wegschaider. Computer Generated Proofs of Binomial Multi-sum Identities, Ph.D. thesis, Research Institute for Symbolic Computation, Institut für Mathematik, Johannes Kepler Universität, Linz, May 1997.

[13] D. Zeilberger. "Proof of the Alternating Sign Matrix Conjecture." Electronic J. Combinatorics 3, No. 2 (1996), R13, pp. 1-84.

[14] D. Zeilberger. "Proof of the Refined Alternating Sign Matrix Conjecture." New York J. Math. 2 (1996), pp. 59-68. 


\section{APPENDIX I (Derivation of the 4-term recursion)}

In this appendix we derive the the 4-term recursion corresponding to the Quadratic Polynomial Riemann hypothesis. We begin with a general framework.

Let $f(x)$ be a positive function, and $g(x)$ a real function, both defined on the unit interval $0<x<1$ and chosen so that the integral

$$
\tilde{g}_{f}(s)=\int_{0}^{1} f(x)^{s} f(1-x)^{1-s} g(x) d^{*} x
$$

is an analytic function of the complex variable $s=\sigma+i t$ on the critical strip $0<\sigma<1$. The measure $d^{*} x$ denotes the invariant measure for a group of transformations

$$
\left[y=m_{\lambda}(x): \lambda>0\right]
$$

of the unit interval to itself, defined for each $\lambda>0$ via

$$
\frac{f(1-y)}{f(y)}=\frac{1}{\lambda} \frac{f(1-x)}{f(x)}
$$

For each such $\lambda$ there is a $\lambda$-multiplier $M_{\lambda}(x)$ defined by

$$
f(y)=M_{\lambda}(x) f(x)
$$

so that

$$
f(1-y)=\frac{M_{\lambda}(x)}{\lambda} f(1-x) .
$$

Of particular interest are those choices of $f$ and $g$ for which $\tilde{g}_{f}(s)$ may be extended to a meromorphic function in the complex plane and there satisfying a functional equation such as

$$
\tilde{g}_{f}(s)=\tilde{g}_{f}(1-s) .
$$

This functional equation would follow, for example, from a choice of $g$ satisfying

$$
g(x)=g(1-x)
$$

and of course a very special interest would obtain in those cases in which $\tilde{g}_{f}(s)$ satisfies, additionally, a Riemann hypothesis; i. e., in those cases of $\tilde{g}_{f}(s)$ whose zeros $\rho=\beta+i \gamma$ in the critical strip satisfy $\beta=\frac{1}{2}$.

We have considered in this paper the special polynomial case, $f(x)=x$, in which

$$
\begin{aligned}
M_{\lambda}(x) & =\frac{\lambda}{1+(\lambda-1) x} \\
m_{\lambda}(x) & =x M_{\lambda}(x) \\
d^{*}(x)= & =\frac{d x}{x(1-x)},
\end{aligned}
$$

together with a sequence, $g_{n}(x)=[x(x-1)+r]^{n}$, of powers of a quadratic. The $g_{n}$ satisfy differential-difference equations, from which it is readily deduced that the sequence, $P_{n}(s, r)=$ $T\left[g_{n}\right]$, satisfy 4 -term recursions. 
To indicate how these recursions are obtained, make the substitution,

$$
y=m_{\lambda}(x)
$$

in the integral,

$$
\tilde{g}_{f}(s)=\int_{0}^{1} f(y)^{s} f(1-y)^{1-s} g(y) d^{*} y
$$

to get

$$
\tilde{g}_{f}(s) \lambda^{1-s}=\int_{0}^{1} f(x)^{s} f(1-x)^{1-s} D(\lambda, x) d^{*} x
$$

where

$$
D(\lambda, x)=M_{\lambda}(x) g\left(m_{\lambda}(x)\right) .
$$

Evaluating the derivatives with respect to $\lambda$ at $\lambda=1$ we get

$$
\begin{aligned}
(1-s) \tilde{g}(s) & =\int_{0}^{1} f(y)^{s} f(1-y)^{1-s} \dot{D}(1, x) d^{*} y \\
s(1-s) \tilde{g}(s) & =\int_{0}^{1} f(y)^{s} f(1-y)^{1-s} \ddot{D}(1, x) d^{*} y
\end{aligned}
$$

In the special (polynomial) case

$$
\begin{aligned}
M_{1} & =1 \\
\left.\frac{d}{d \lambda} M(\lambda)\right|_{\lambda=1} & =1-x \\
\left.\frac{d^{2}}{d \lambda^{2}} M(\lambda)\right|_{\lambda=1} & =x(x-1) \\
\left.\frac{d}{d \lambda} m(\lambda)\right|_{\lambda=1} & =x(1-x) \\
\left.\frac{d^{2}}{d \lambda^{2}} m(\lambda)\right|_{\lambda=1} & =x^{2}(x-1)
\end{aligned}
$$

and

$$
\left.\frac{d^{2}}{d \lambda^{2}} D(\lambda, x)\right|_{\lambda=1}=x^{2}(1-x)^{2} \ddot{g}_{n}(x)+x(x-1)(2 x-1) \dot{g}_{n}(x)+x(x-1) g_{n}(x)
$$

and the recursion (5) given in section 1 follows directly from

$$
\begin{aligned}
g_{n} & =g_{1}^{n} \\
\dot{g}_{n} & =n(2 x-1) g_{1}^{n-1} \\
\ddot{g}_{n} & =n(n-1)(2 x-1)^{2} g_{1}^{n-2}+2 n g_{1}^{n-1} \\
(2 x-1)^{2} & =4\left(g_{1}-r\right)+1 \\
x(x-1) & =g_{1}-r .
\end{aligned}
$$

We remark that the same method will produce higher order recursions for the $T$-transforms of sequences of powers $Q(x)^{n}$. 


\section{APPENDIX II (Renormalized 4-term recursion)}

Renormalization is an attempt to see what is happening in the $P_{n}$-recursion (5) of section 1 for large $n$. As a first step we put $s=\frac{1}{2}+i t$, where $t$ is a real variable. The real polynomials

$$
P_{n}\left(\frac{1}{2}+i t\right)
$$

are polynomials in $u=-t^{2}$ and we put

$$
p_{n}(u)=P_{n}\left(\frac{1}{2}+i t\right)
$$

The $p_{n}$ satisfy the recursion

$$
\begin{aligned}
(2 n+2)(2 n+1) p_{n+1}(u) & =\left[-\frac{1}{4}+u+12 r n^{2}+8 r n+2 r-n^{2}-n\right] p_{n}(u) \\
& -\left[12 r^{2} n^{2}-2 r n^{2}-2 r^{2} n\right] p_{n-1}(u) \\
& +\left[n(n-1)\left(4 r^{3}-r^{2}\right)\right] p_{n-2}(u) .
\end{aligned}
$$

Next, divide the recursion by $n^{2}$ and throw away the terms in the coefficients that go to zero as $\mathrm{n}$ goes to infinity. There results a recursion

$$
4 e_{n+1}(u)=\left(\frac{u}{n^{2}}+12 r-1\right) e_{n}(u)-\left(12 r^{2}-2 r\right) e_{n-1}(u)+\left(4 r^{3}-r^{2}\right) e_{n-2}(u) .
$$

Next, multiply this recursion by $4^{n}$ and put $f_{n}=4^{n} e_{n}$ to get

$$
f_{n+1}(u)=\left(\frac{u}{n^{2}}+12 r-1\right) f_{n}(u)-8 r(6 r-1) f_{n-1}(u)+16 r^{2}(4 r-1) f_{n-2}(u) .
$$

Next, put $u=n^{2} v$ to get a recursion

$$
\begin{aligned}
g_{n+1}\left(\left(\frac{n}{n+1}\right)^{2} v\right) & =(v+12 r-1) g_{n}(v) \\
& -8 r(6 r-1) g_{n-1}\left(\left(\frac{n}{n-1}\right)^{2} v\right) \\
+ & 16 r^{2}(4 r-1) g_{n-2}\left(\left(\frac{n}{n-2}\right)^{2} v\right) .
\end{aligned}
$$

We replace this recursion with

$$
h_{n+1}(v)=(v+12 r-1) h_{n}(v)-8 r(6 r-1) h_{n-1}(v)+16 r^{2}(4 r-1) h_{n-2}(v) .
$$

Next, put $v+12 r-1=u$ to get a recursion

$$
I_{n+1}(u)=u I_{n}(u)-A I_{n-1}(u)+B I_{n-2}(u)
$$

with

$$
\begin{aligned}
& A=8 r(6 r-1) \\
& B=16 r^{2}(4 r-1) .
\end{aligned}
$$


Finally, if we put

$$
\begin{aligned}
u & =x B^{\frac{1}{3}} \\
A & =C B^{\frac{2}{3}} \\
Q_{n}(x) & =B^{-\frac{n}{3}} I_{n}\left(x B^{\frac{1}{3}}\right)
\end{aligned}
$$

then we get a polynomial recursion

$$
Q_{n}(x)=x Q_{n-1}(x)-C Q_{n-2}(x)+Q_{n-3}(x)
$$

with

$$
C=\frac{8 r(6 r-1)}{\left[16 r^{2}(4 r-1)\right]^{\frac{2}{3}}} .
$$

In the recursion (44), we considered the range $\frac{1}{4}<r<\infty$, which is converted to the range $\infty>C>3$. This is how the critical value $C=3$ emerges from the calculations.

From the asymptotic recursion (45), we obtain a sequence of polynomials, the first five of which are given by

$$
\begin{aligned}
& Q_{0}(x, C)=1 \\
& Q_{1}(x, C)=x \\
& Q_{2}(x, C)=x^{2}-C \\
& Q_{3}(x, C)=x^{3}-2 C x+1 \\
& Q_{4}(x, C)=x^{4}-3 C x^{2}+2 x+C^{2}
\end{aligned}
$$

We note that if a sequence of polynomials $q_{n}$ is defined by

$$
\begin{aligned}
& q_{0}(x, C)=1 \\
& q_{1}(x, C)=x \\
& q_{2}(x, C)=x^{2}-C
\end{aligned}
$$

and thereafter by the recursion

$$
q_{n}(x)=x q_{n-1}(x)-C q_{n-2}(x)-q_{n-3}(x),
$$

then the $q_{n}$ are related to the $Q_{n}$ by

$$
q_{n}(x)=(-1)^{n} Q_{n}(-x) .
$$

It follows that $Q_{n}$ has real zeros for some pair $n$ and $C$, if and only if $q_{n}$ has real zeros. In this paper we preferred to work with the second recursion, the one with the minus sign, because the moments that are defined by this sequence are non-negative, while the corresponding moments defined by the first recursion alternate in sign. 